Supporting Information for

\title{
Triterpenoid Saponins in Oat Bran and Their Levels in Commercial Oat
}

\section{Products}

Changling Hu and Shengmin Sang*

Laboratory for Functional Foods and Human Health, Center for Excellence in PostHarvest Technologies, North Carolina Agricultural and Technical State University, North Carolina Research Campus, 500 Laureate Way, Kannapolis, North Carolina 28081, United States

\section{Corresponding author:}

Shengmin Sang, Ph.D.

North Carolina Agricultural and Technical State University

North Carolina Research Campus

Tel: 704-250-5710; Fax: 704-250-5709

Email: ssang@ncat.edu or shengminsang@yahoo.com 


\section{Contents}

Table S1. ${ }^{1} \mathrm{H}-\mathrm{NMR}$ Data of sugar moieties of compounds $1-4$ and 7-8 ( $\delta$ in ppm and $J$ in $\left.\mathrm{Hz}\right)^{\mathrm{a}} 1$ Table S2. ${ }^{13} \mathrm{C}$-NMR Data of sugar moieties of compounds $1-4$ and $7-8(\delta \text { in ppm })^{\mathrm{a}} \ldots \ldots \ldots \ldots \ldots \ldots . . . . . . .2$ Table S3. The standard curves, limits of detection (LOD) and quantification (LOQ), interday and intraday precision of the four triterpenoid saponin standards, and coverage of the triterpenoid saponin compounds by each standard........................................................................

Compound 1: ${ }^{1} \mathrm{H}$ NMR spectrum $\left(600 \mathrm{MHz}, \mathrm{CD}_{3} \mathrm{OD}\right)$....................................................................

Compound 1: ${ }^{1} \mathrm{H}$ NMR spectrum $\left(600 \mathrm{MHz}, \mathrm{CD}_{3} \mathrm{OD}\right)$-expansion....................................................

Compound 1: ${ }^{1} \mathrm{H}$ NMR spectrum $\left(600 \mathrm{MHz}, \mathrm{CD}_{3} \mathrm{OD}\right)$-expansion.....................................................6

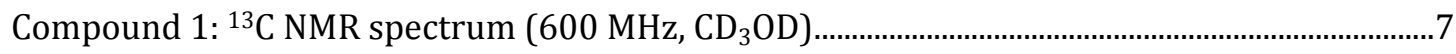

Compound $1:{ }^{13} \mathrm{C}$ NMR spectrum $\left(600 \mathrm{MHz}, \mathrm{CD}_{3} \mathrm{OD}\right.$ )-expansion.................................................

Compound $1:{ }^{13} \mathrm{C}$ NMR spectrum $\left(600 \mathrm{MHz}, \mathrm{CD}_{3} \mathrm{OD}\right.$ )-expansion...............................................

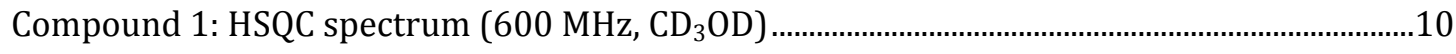

Compound $1:{ }^{1} \mathrm{H}-{ }^{1} \mathrm{H}$ COSY spectrum $\left(600 \mathrm{MHz}, \mathrm{CD}_{3} \mathrm{OD}\right)$........................................................11

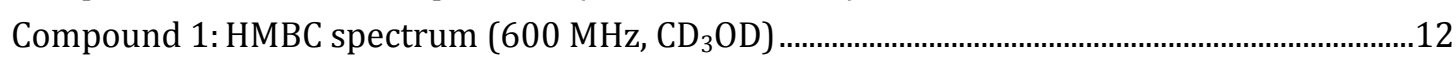

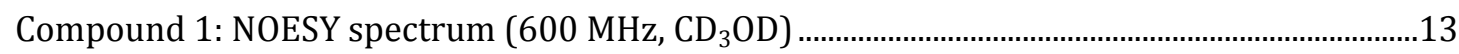

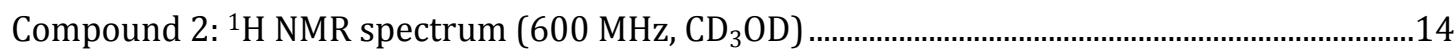

Compound 2: ${ }^{1} \mathrm{H}$ NMR spectrum $\left(600 \mathrm{MHz}, \mathrm{CD}_{3} \mathrm{OD}\right)$-expansion .................................................15

Compound 2: ${ }^{1} \mathrm{H}$ NMR spectrum $\left(600 \mathrm{MHz}, \mathrm{CD}_{3} \mathrm{OD}\right)$-expansion …................................................16

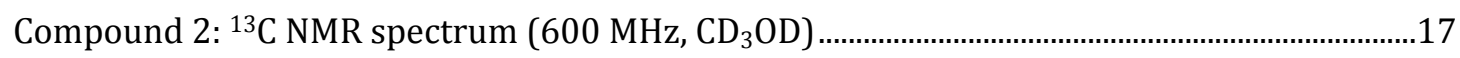

Compound 2: ${ }^{13} \mathrm{C}$ NMR spectrum $\left(600 \mathrm{MHz}, \mathrm{CD}_{3} \mathrm{OD}\right.$ )-expansion.................................................18

Compound $2:{ }^{13} \mathrm{C}$ NMR spectrum $\left(600 \mathrm{MHz}, \mathrm{CD}_{3} \mathrm{OD}\right.$ )-expansion..................................................19

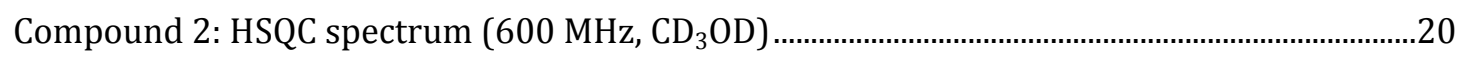

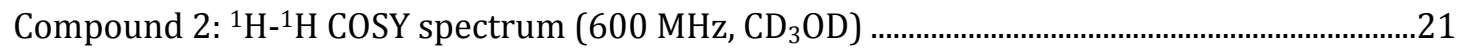

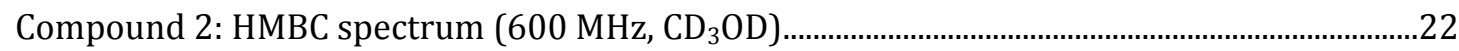

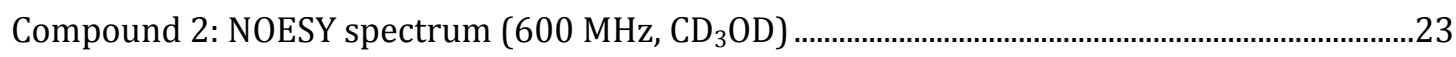

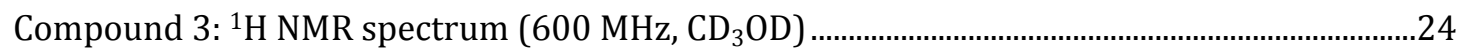

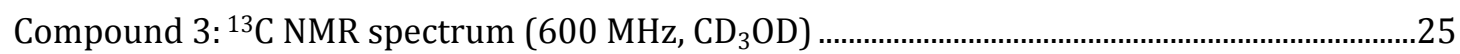

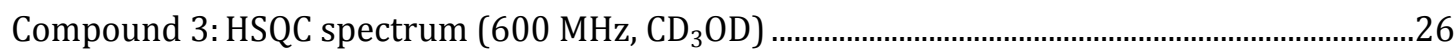

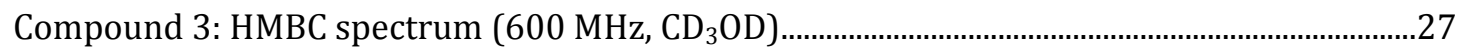

Compound 3: NOESY spectrum $\left(600 \mathrm{MHz}, \mathrm{CD}_{3} \mathrm{OD}\right)$......................................................................28

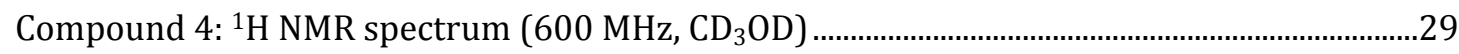

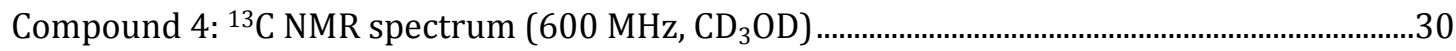

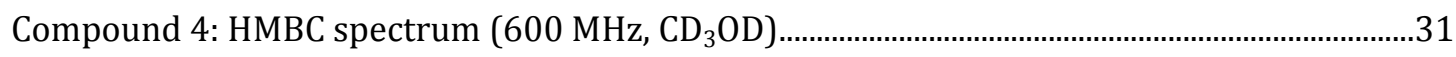

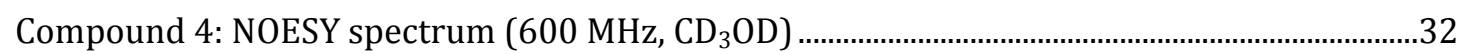

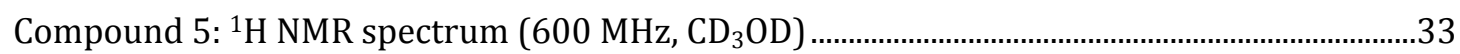

Compound 6: ${ }^{1} \mathrm{H}$ NMR spectrum $\left(600 \mathrm{MHz}, \mathrm{CD}_{3} \mathrm{OD}\right)$..................................................................

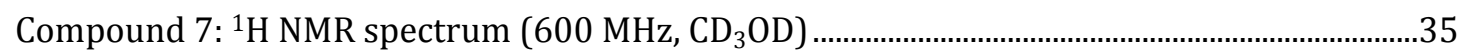

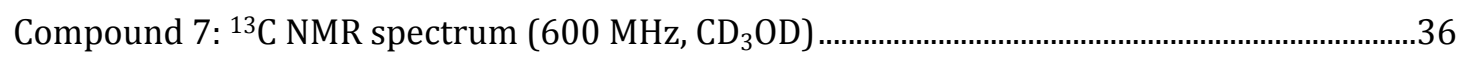

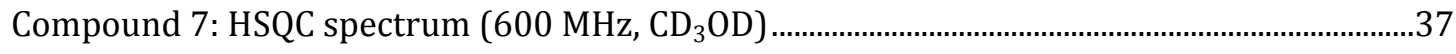

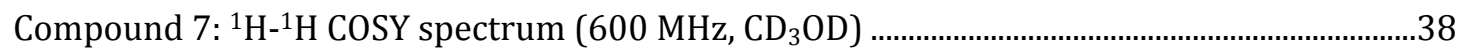

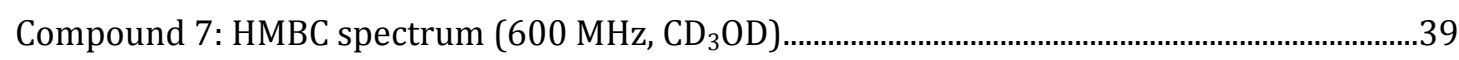

Compound 7: NOESY spectrum (600 MHz, $\left.\mathrm{CD}_{3} \mathrm{OD}\right)$..................................................................40

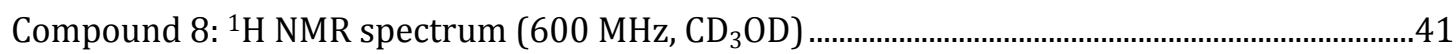


Compound 8: ${ }^{13} \mathrm{C}$ NMR spectrum $\left(600 \mathrm{MHz}, \mathrm{CD}_{3} \mathrm{OD}\right)$ . .42

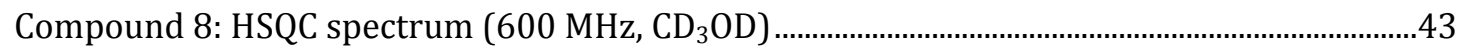

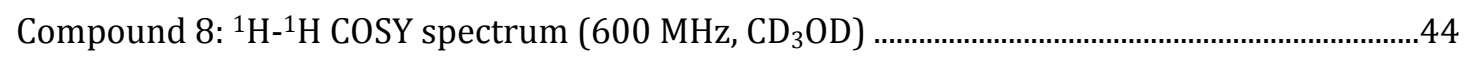

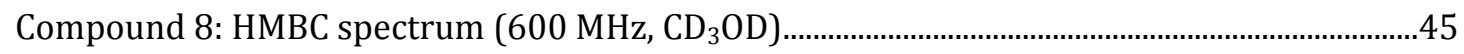

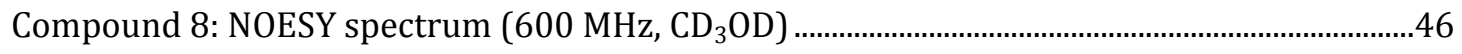


Table S1. ${ }^{1} \mathrm{H}-\mathrm{NMR}$ Data of sugar moieties of compounds $1-4$ and $7-8(\delta \text { in ppm and } J \text { in } \mathrm{Hz})^{\mathrm{a}}$

\begin{tabular}{|c|c|c|c|c|c|c|}
\hline No & 1 & 2 & 3 & 4 & 7 & 8 \\
\hline $1 "$ & $4.53(\mathrm{~d}, 5.2)$ & $4.54(\mathrm{~d}, 5.2)$ & $4.54(\mathrm{~d}, 5.2)$ & $4.54(\mathrm{~d}, 5.2)$ & $4.53(\mathrm{~d}, 5.2)$ & $4.53(\mathrm{~d}, 5.2)$ \\
\hline $2 "$ & $3.86(\mathrm{dd}, 7.8,5.2)$ & $3.87(\mathrm{dd}, 7.8,5.2)$ & $3.87(\mathrm{dd}, 7.8,5.2)$ & $3.88(\mathrm{dd}, 7.8,5.2)$ & $3.87(\mathrm{dd}, 7.8,5.2)$ & $3.86(\mathrm{dd}, 7.8,5.2)$ \\
\hline $3 "$ & 3.66(overlapped) & 3.68(overlapped) & 3.66(overlapped) & 3.65(overlapped) & 3.65 (overlapped) & 3.65 (overlapped) \\
\hline $4 "$ & $3.30(\mathrm{~m})$ & $3.30(\mathrm{~m})$ & $3.30(\mathrm{~m})$ & $3.30(\mathrm{~m})$ & $3.32(\mathrm{~m})$ & $3.30(\mathrm{~m})$ \\
\hline \multirow[t]{2}{*}{$5^{\prime \prime}$} & 4.14(overlapped) & 4.19(overlapped) & 4.14(overlapped) & 4.13(overlapped) & 4.14(overlapped) & 4.14(overlapped) \\
\hline & 3.55 (overlapped) & 3.55(overlapped) & 3.55(overlapped) & 3.56(overlapped) & 3.56 (overlapped) & 3.56 (overlapped) \\
\hline $1 " '$ & $4.63(\mathrm{~d}, 7.8)$ & $4.63(\mathrm{~d}, 7.8)$ & $4.63(\mathrm{~d}, 7.8)$ & $4.63(\mathrm{~d}, 7.8)$ & $4.62(\mathrm{~d}, 7.8)$ & $4.62(\mathrm{~d}, 7.8)$ \\
\hline $2^{\prime \prime \prime}$ & 3.22(overlapped) & 3.22(overlapped) & 3.22(overlapped) & 3.21(overlapped) & 3.24(overlapped) & 3.21 (overlapped) \\
\hline $3^{\prime \prime \prime}$ & 3.26 (overlapped) & 3.26 (overlapped) & 3.26(overlapped) & 3.25(overlapped) & 3.26 (overlapped) & 3.24 (overlapped) \\
\hline 4"' & 3.28(overlapped) & 3.28(overlapped) & 3.28(overlapped) & 3.28(overlapped) & 3.30 (overlapped) & 3.30 (overlapped) \\
\hline $5^{\prime \prime \prime}$ & $3.82(\mathrm{~m})$ & $3.83(\mathrm{~m})$ & $3.83(\mathrm{~m})$ & $3.82(\mathrm{~m})$ & $3.84(\mathrm{~m})$ & $3.84(\mathrm{~m})$ \\
\hline \multirow[t]{2}{*}{ 6"' } & 3.75(overlapped) & 3.76 (overlapped) & 3.76 (overlapped) & 3.74(overlapped) & 3.74(overlapped) & 3.74(overlapped) \\
\hline & 3.60 (overlapped) & 3.60 (overlapped) & 3.60(overlapped) & 3.59(overlapped) & 3.58 (overlapped) & 3.58(overlapped) \\
\hline $1^{\prime \prime \prime \prime}$ & $4.47(\mathrm{~d}, 7.8)$ & $4.46(\mathrm{~d}, 7.8)$ & $4.46(\mathrm{~d}, 7.8)$ & $4.47(\mathrm{~d}, 7.8)$ & $4.47(\mathrm{~d}, 7.8)$ & $4.47(\mathrm{~d}, 7.8)$ \\
\hline $2^{\prime \prime \prime \prime}$ & 3.25(overlapped) & 3.24(overlapped) & 3.25(overlapped) & 3.25 (overlapped) & 3.23(overlapped) & 3.25 (overlapped) \\
\hline 3 '"' & 3.88(overlapped) & 3.86 (overlapped) & 3.86(overlapped) & 3.88 (overlapped) & 3.80 (overlapped) & 3.85 (overlapped) \\
\hline 4"'" & 3.60(overlapped) & 3.60 (overlapped) & 3.60(overlapped) & 3.60 (overlapped) & 3.60 (overlapped) & 3.60 (overlapped) \\
\hline $5^{\prime \prime \prime \prime}$ & $3.37(\mathrm{~m})$ & $3.37(\mathrm{~m})$ & $3.37(\mathrm{~m})$ & $3.36(\mathrm{~m})$ & $3.35(\mathrm{~m})$ & $3.36(\mathrm{~m})$ \\
\hline \multirow[t]{2}{*}{$6^{\prime \prime \prime \prime}$} & 3.96(overlapped) & 3.92(overlapped) & 3.96 (overlapped) & 3.86(overlapped) & 3.86 (overlapped) & 3.84(overlapped) \\
\hline & 3.62(overlapped) & 3.62(overlapped) & 3.62(overlapped) & 3.62(overlapped) & 3.62(overlapped) & 3.64(overlapped) \\
\hline
\end{tabular}

${ }^{a}$ Assignments were made by a combination of 1D and 2D NMR experiments. 
Table S2. ${ }^{13} \mathrm{C}-\mathrm{NMR}$ Data of sugar moieties of compounds $1-4$ and $7-8\left(\delta\right.$ in ppm) ${ }^{\mathrm{a}}$

\begin{tabular}{|c|c|c|c|c|c|c|}
\hline No & 1 & 2 & 3 & 4 & 7 & 8 \\
\hline 1" & $104.5 \mathrm{~d}$ & $104.5 \mathrm{~d}$ & $104.5 \mathrm{~d}$ & $104.5 \mathrm{~d}$ & $104.5 \mathrm{~d}$ & $104.5 \mathrm{~d}$ \\
\hline $2^{\prime \prime}$ & $79.1 \mathrm{~d}$ & $79.1 \mathrm{~d}$ & $79.0 \mathrm{~d}$ & $79.0 \mathrm{~d}$ & $79.0 \mathrm{~d}$ & $79.0 \mathrm{~d}$ \\
\hline 3" & $78.1 \mathrm{~d}$ & $78.1 \mathrm{~d}$ & $78.0 \mathrm{~d}$ & $78.1 \mathrm{~d}$ & $78.1 \mathrm{~d}$ & $78.1 \mathrm{~d}$ \\
\hline 4" & $71.5 \mathrm{~d}$ & $71.5 \mathrm{~d}$ & $71.4 \mathrm{~d}$ & $71.5 \mathrm{~d}$ & $71.5 \mathrm{~d}$ & $71.5 \mathrm{~d}$ \\
\hline $5^{\prime \prime}$ & $64.5 \mathrm{t}$ & $64.5 \mathrm{t}$ & $63.1 \mathrm{t}$ & $64.6 \mathrm{t}$ & $64.6 \mathrm{t}$ & $64.9 \mathrm{t}$ \\
\hline 1"' & $104.4 \mathrm{~d}$ & $104.4 \mathrm{~d}$ & $104.4 \mathrm{~d}$ & $104.4 \mathrm{~d}$ & $104.4 \mathrm{~d}$ & $104.4 \mathrm{~d}$ \\
\hline $2^{\prime \prime \prime}$ & $71.8 \mathrm{~d}$ & $71.8 \mathrm{~d}$ & $71.8 \mathrm{~d}$ & $71.8 \mathrm{~d}$ & $71.8 \mathrm{~d}$ & $71.8 \mathrm{~d}$ \\
\hline 3"' & $75.5 \mathrm{~d}$ & $75.5 \mathrm{~d}$ & $75.8 \mathrm{~d}$ & $76.3 \mathrm{~d}$ & $76.3 \mathrm{~d}$ & $75.8 \mathrm{~d}$ \\
\hline 4"' & $78.3 \mathrm{~d}$ & $78.3 \mathrm{~d}$ & $78.2 \mathrm{~d}$ & $78.2 \mathrm{~d}$ & $78.2 \mathrm{~d}$ & $78.2 \mathrm{~d}$ \\
\hline $5^{\prime \prime \prime}$ & $77.9 \mathrm{~d}$ & $77.9 \mathrm{~d}$ & $78.0 \mathrm{~d}$ & $77.9 \mathrm{~d}$ & $77.9 \mathrm{~d}$ & $78.0 \mathrm{~d}$ \\
\hline 6"' & $62.7 \mathrm{t}$ & $63.1 \mathrm{t}$ & $62.7 \mathrm{t}$ & $62.8 \mathrm{t}$ & $62.8 \mathrm{t}$ & $62.7 \mathrm{t}$ \\
\hline $1^{\prime \prime \prime \prime}$ & $105.8 \mathrm{~d}$ & $105.8 \mathrm{~d}$ & $105.8 \mathrm{~d}$ & $105.8 \mathrm{~d}$ & $105.8 \mathrm{~d}$ & $105.9 \mathrm{~d}$ \\
\hline $2^{\prime \prime \prime \prime}$ & $75.0 \mathrm{~d}$ & $75.0 \mathrm{~d}$ & $75.7 \mathrm{~d}$ & $75.5 \mathrm{~d}$ & $75.5 \mathrm{~d}$ & $75.7 \mathrm{~d}$ \\
\hline 3"'" & $78.0 \mathrm{~d}$ & $78.0 \mathrm{~d}$ & $77.9 \mathrm{~d}$ & $78.1 \mathrm{~d}$ & $78.1 \mathrm{~d}$ & $77.9 \mathrm{~d}$ \\
\hline 4"'" & $73.6 \mathrm{~d}$ & $73.6 \mathrm{~d}$ & $73.6 \mathrm{~d}$ & $73.7 \mathrm{~d}$ & $73.7 \mathrm{~d}$ & $73.6 \mathrm{~d}$ \\
\hline $5^{\prime \prime \prime \prime}$ & $78.4 \mathrm{~d}$ & $78.4 \mathrm{~d}$ & $78.1 \mathrm{~d}$ & $78.5 \mathrm{~d}$ & $78.5 \mathrm{~d}$ & $78.1 \mathrm{~d}$ \\
\hline 6"'" & $63.1 \mathrm{t}$ & $62.7 \mathrm{t}$ & $62.8 \mathrm{t}$ & $63.1 \mathrm{t}$ & $63.1 \mathrm{t}$ & $63.1 \mathrm{t}$ \\
\hline
\end{tabular}

${ }^{a}$ Assignments were made by a combination of 1D and 2D NMR experiments. 
Table S3. The standard curves, limits of detection (LOD) and quantification (LOQ), intra- and inter-day precision of the four triterpenoid saponin standards, and coverage of the triterpenoid saponin compounds by each standard

\begin{tabular}{|c|c|c|c|c|c|c|c|}
\hline Analytes & Standard curves & $R^{2}$ & LOD (nM) & LOQ (nM) & $\begin{array}{l}\text { Intra-day precision } \\
\text { (\%) }\end{array}$ & $\begin{array}{l}\text { Inter-day precision } \\
\text { (\%) }\end{array}$ & Coverage \\
\hline 2 & $y=0.0011 x-0.0047$ & 0.9970 & 0.62 & 1.27 & 1.56 & 0.87 & $2,3,6,7,9$ \\
\hline 4 & $y=0.0006 x-0.0035$ & 0.9987 & 0.96 & 2.07 & 2.22 & 1.09 & 4,5 \\
\hline 8 & $y=0.0032 x-0.0037$ & 0.9984 & 0.22 & 0.38 & 0.98 & 0.56 & 8 \\
\hline 10 & $y=0.0004 x-0.0002$ & 0.9994 & 0.065 & 0.32 & 0.79 & 0.42 & $1,10-13$ \\
\hline
\end{tabular}


Compound 1: ${ }^{1} \mathrm{H}$ NMR spectrum (600 MHz, $\left.\mathrm{CD}_{3} \mathrm{OD}\right)$

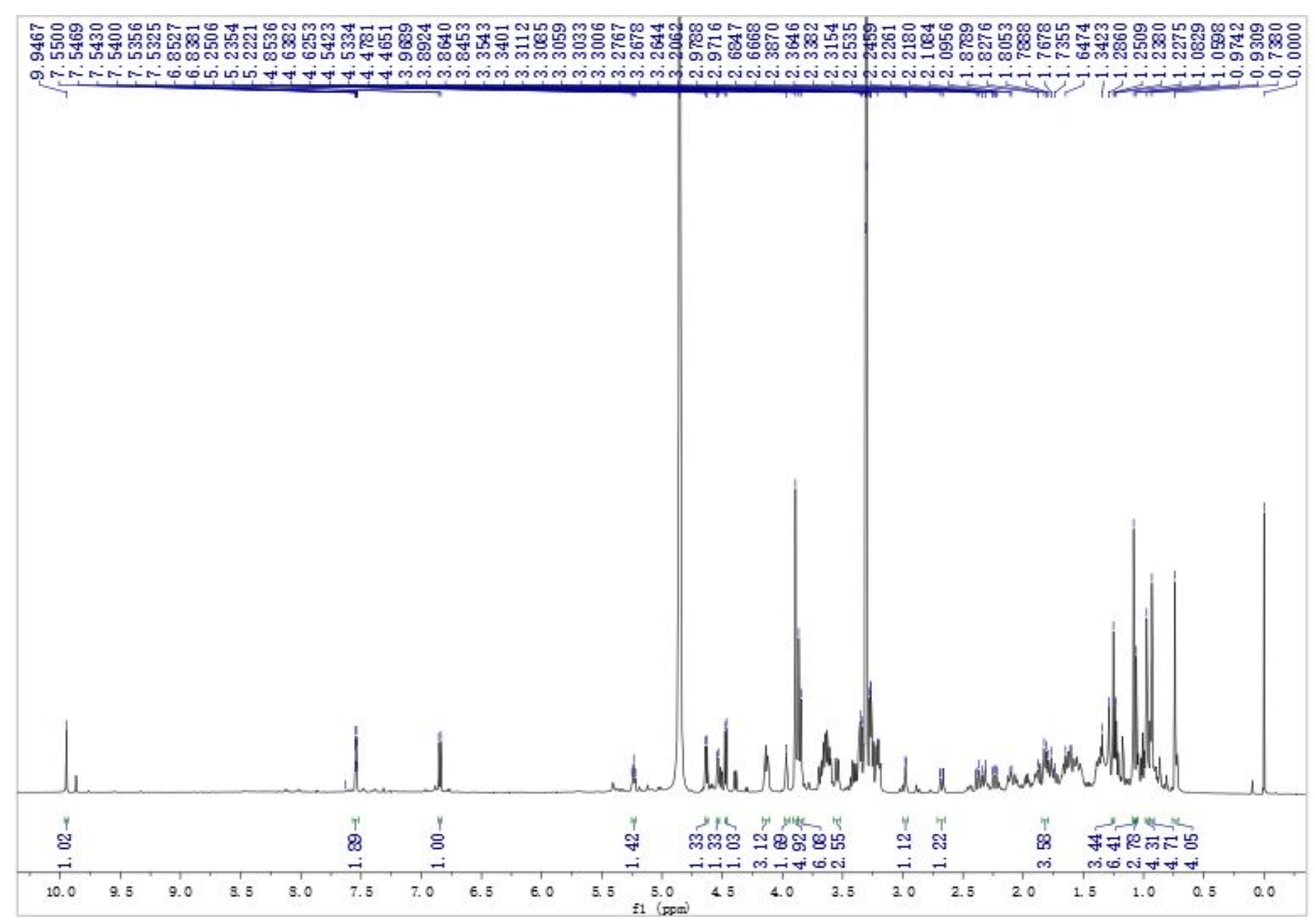


Compound 1: ${ }^{1} \mathrm{H}$ NMR spectrum (600 MHz, $\mathrm{CD}_{3} \mathrm{OD}$ )-expansion

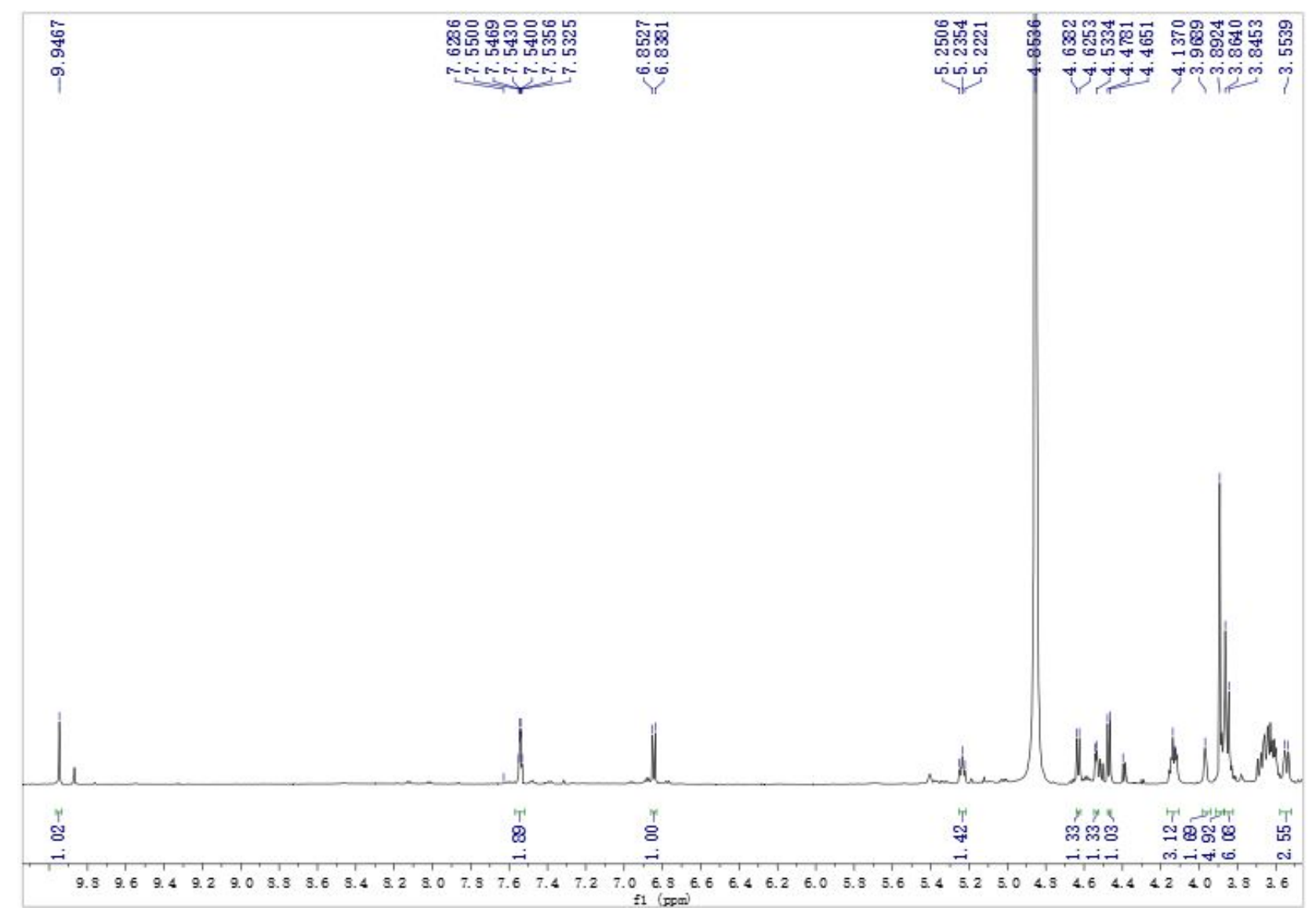


Compound 1: ${ }^{1} \mathrm{H}$ NMR spectrum (600 MHz, $\mathrm{CD}_{3} \mathrm{OD}$ )-expansion

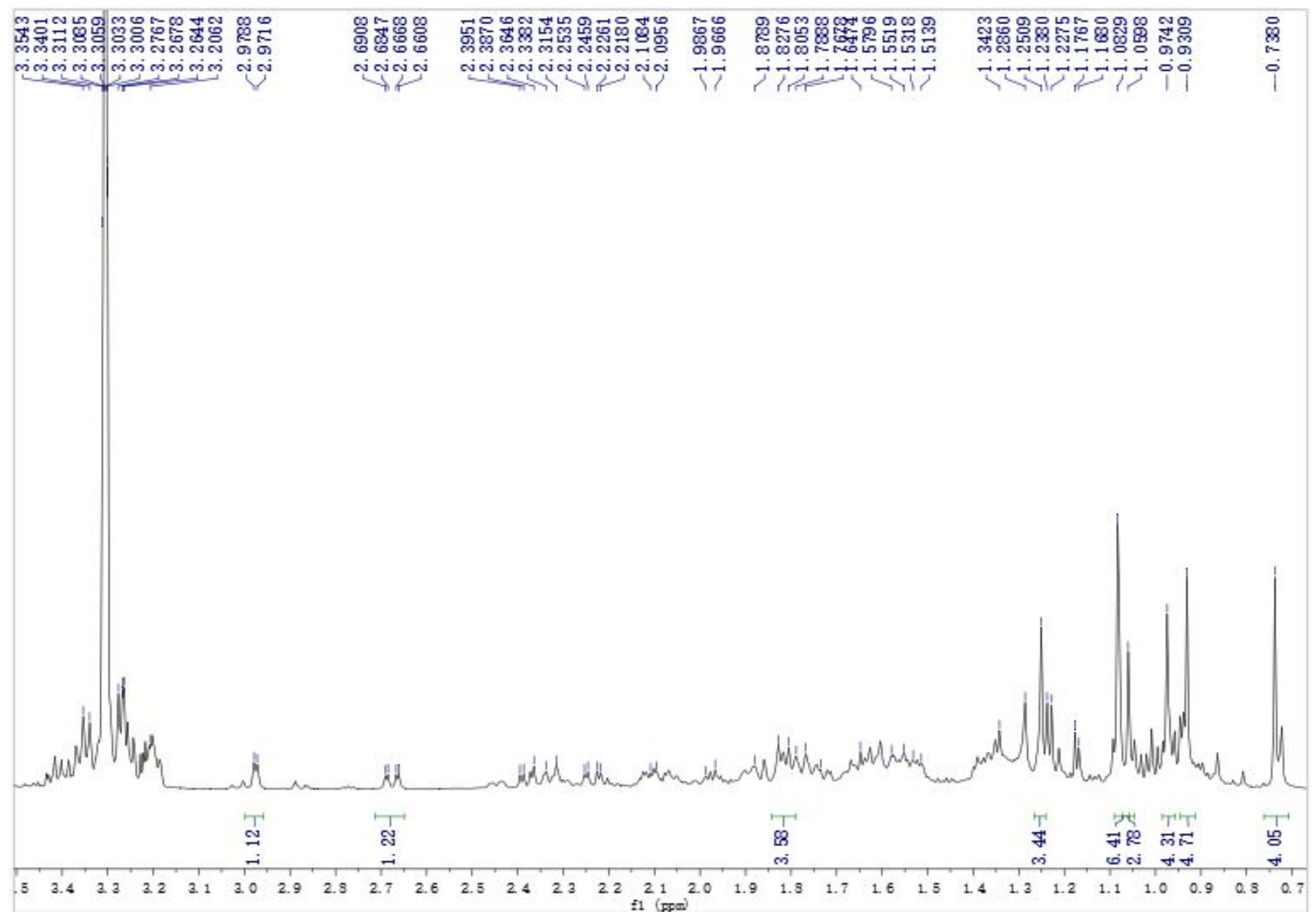


Compound 1: ${ }^{13} \mathrm{C}$ NMR spectrum $\left(600 \mathrm{MHz}, \mathrm{CD}_{3} \mathrm{OD}\right)$

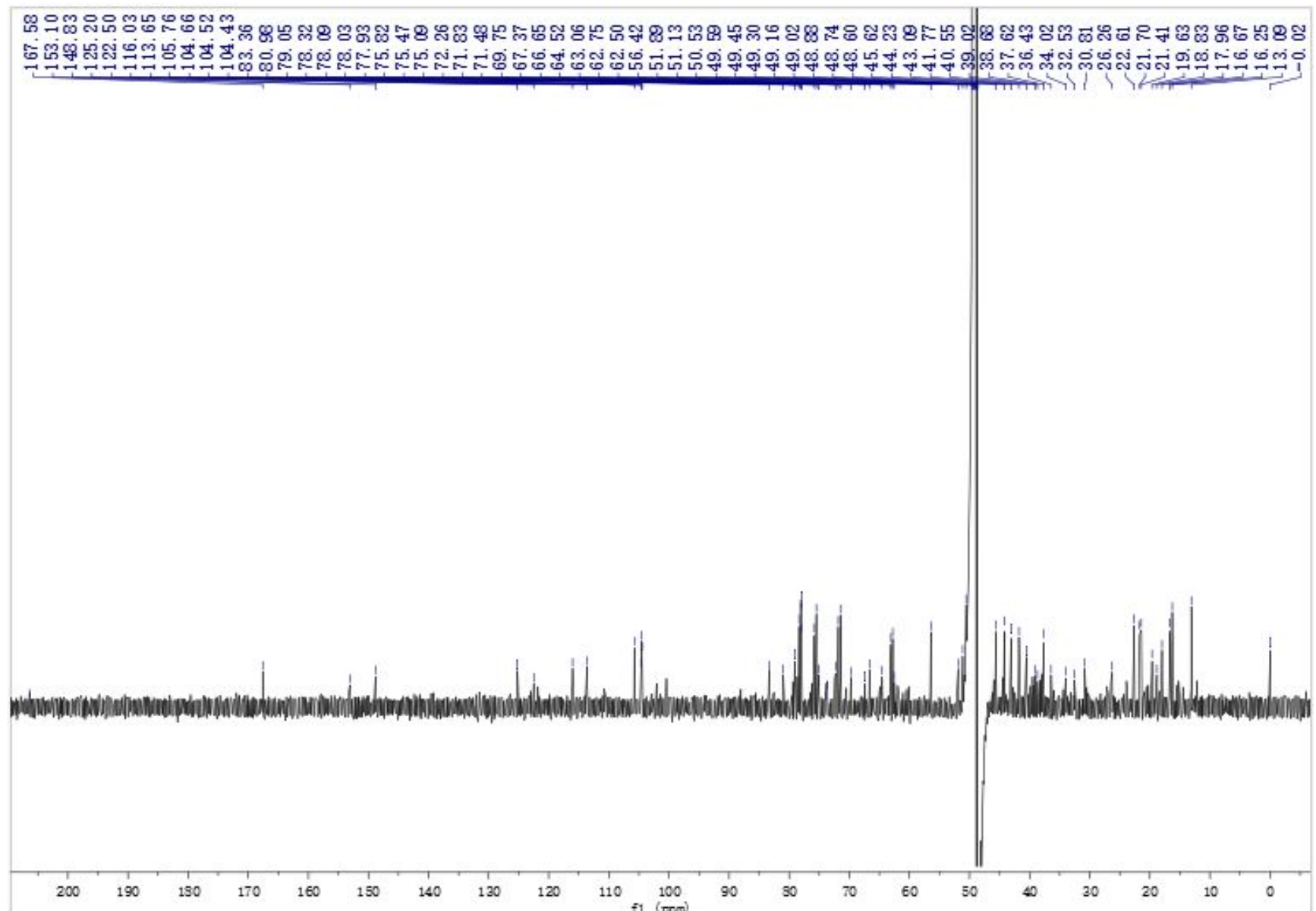


Compound 1: ${ }^{13} \mathrm{C}$ NMR spectrum (600 MHz, $\left.\mathrm{CD}_{3} \mathrm{OD}\right)$-expansion

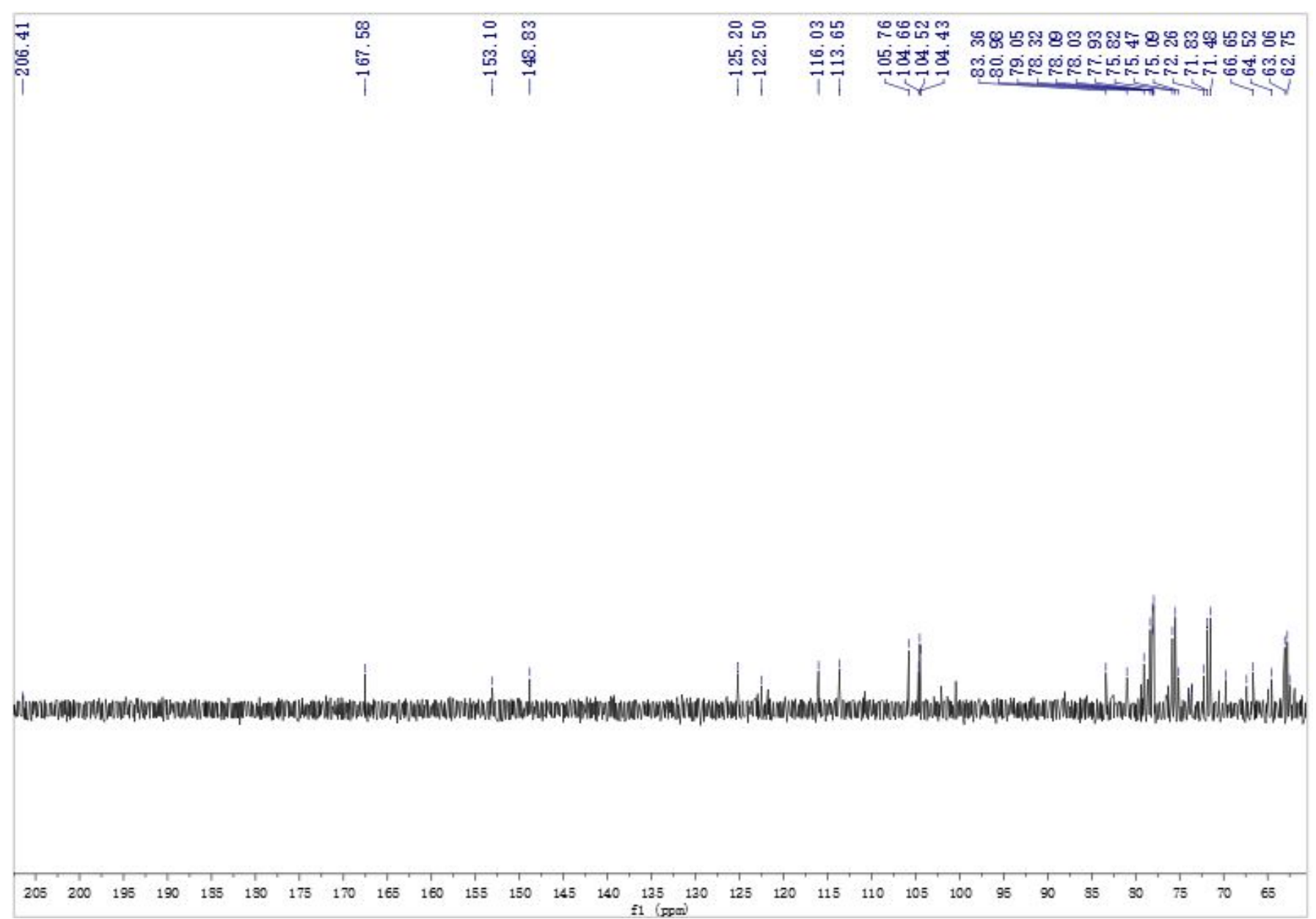


Compound 1: ${ }^{13} \mathrm{C}$ NMR spectrum (600 MHz, $\left.\mathrm{CD}_{3} \mathrm{OD}\right)$-expansion

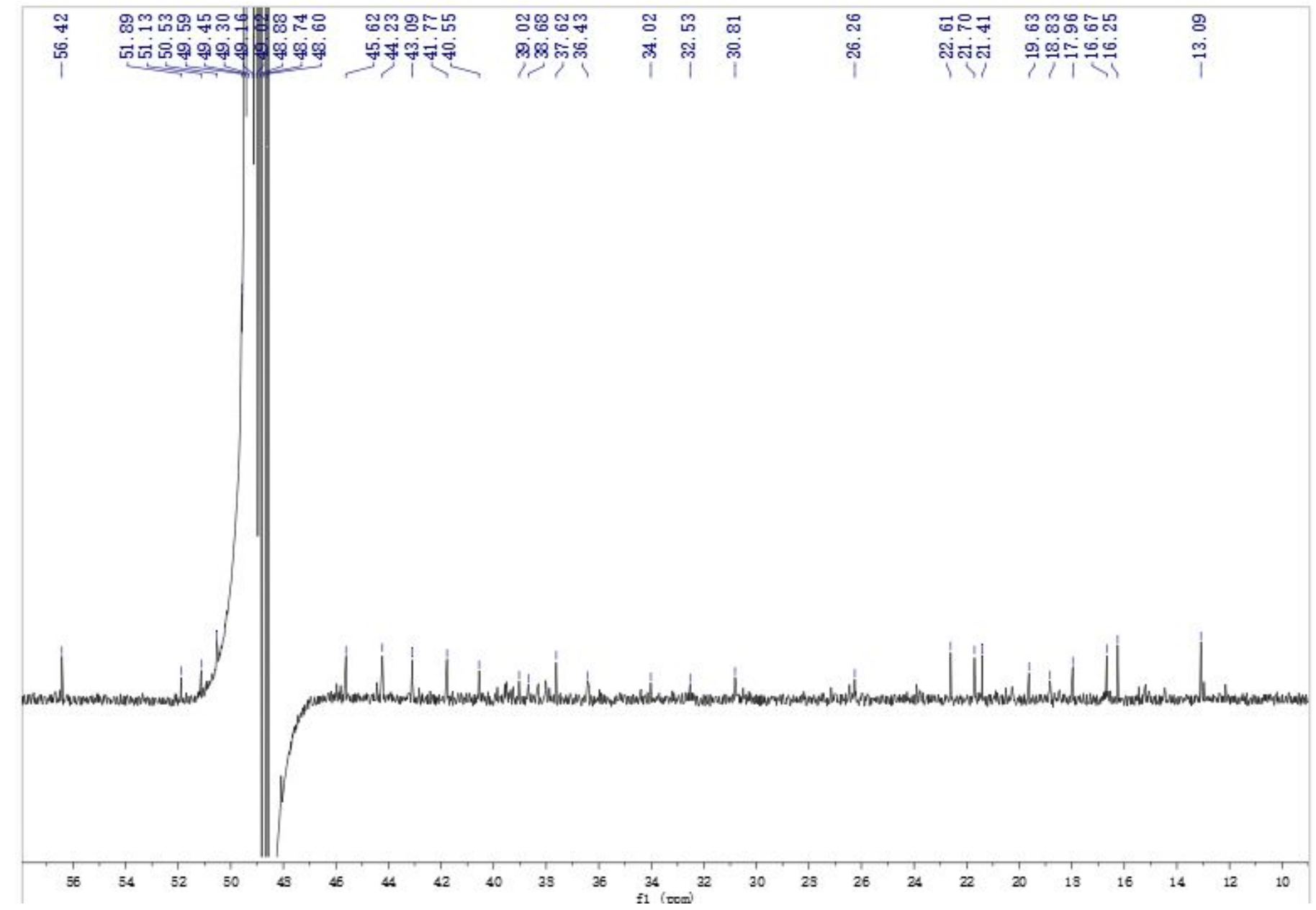


Compound 1: HSQC spectrum (600 MHz, $\left.\mathrm{CD}_{3} \mathrm{OD}\right)$

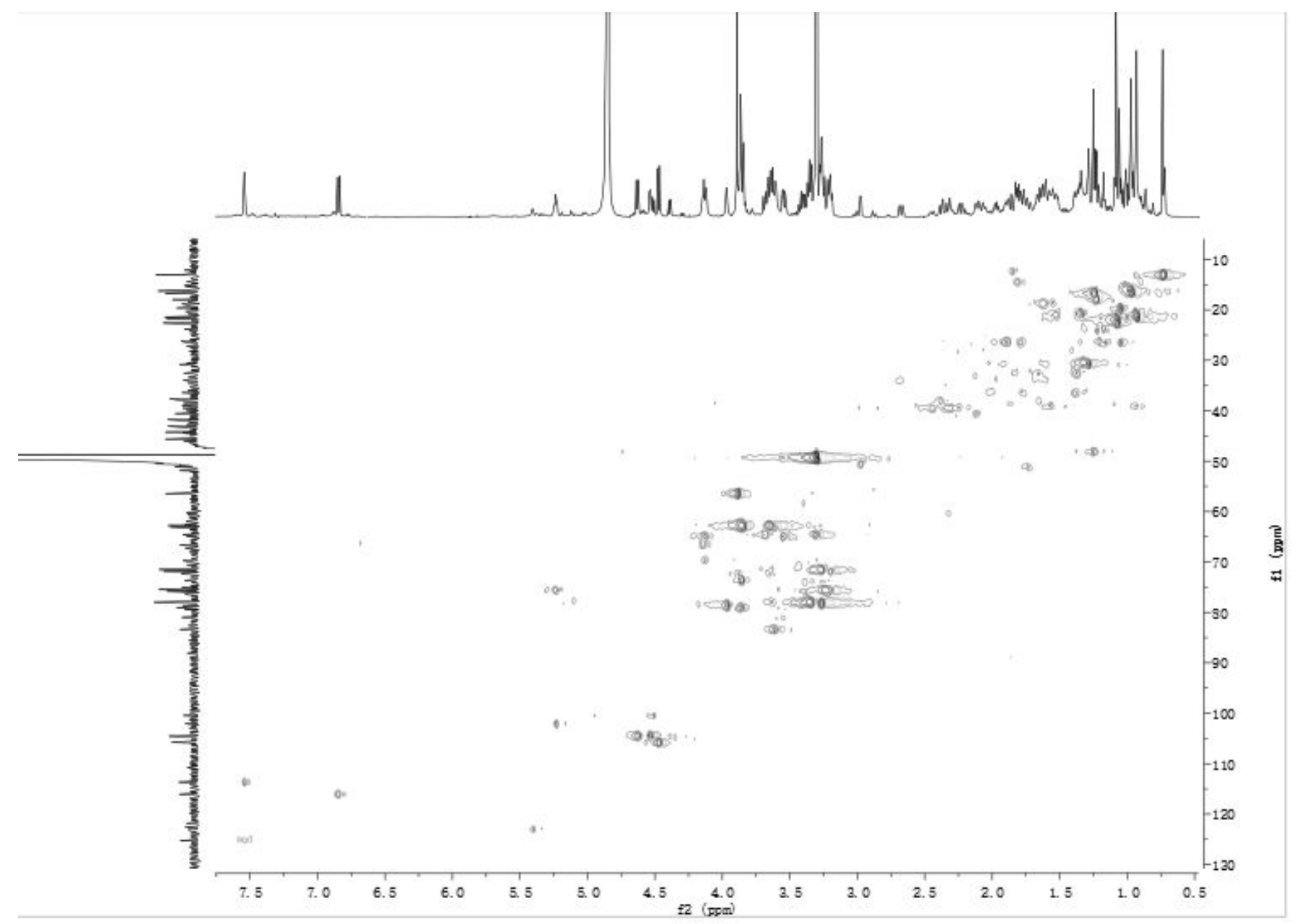


Compound 1: ${ }^{1} \mathrm{H}-{ }^{1} \mathrm{H}$ COSY spectrum $\left(600 \mathrm{MHz}, \mathrm{CD}_{3} \mathrm{OD}\right)$

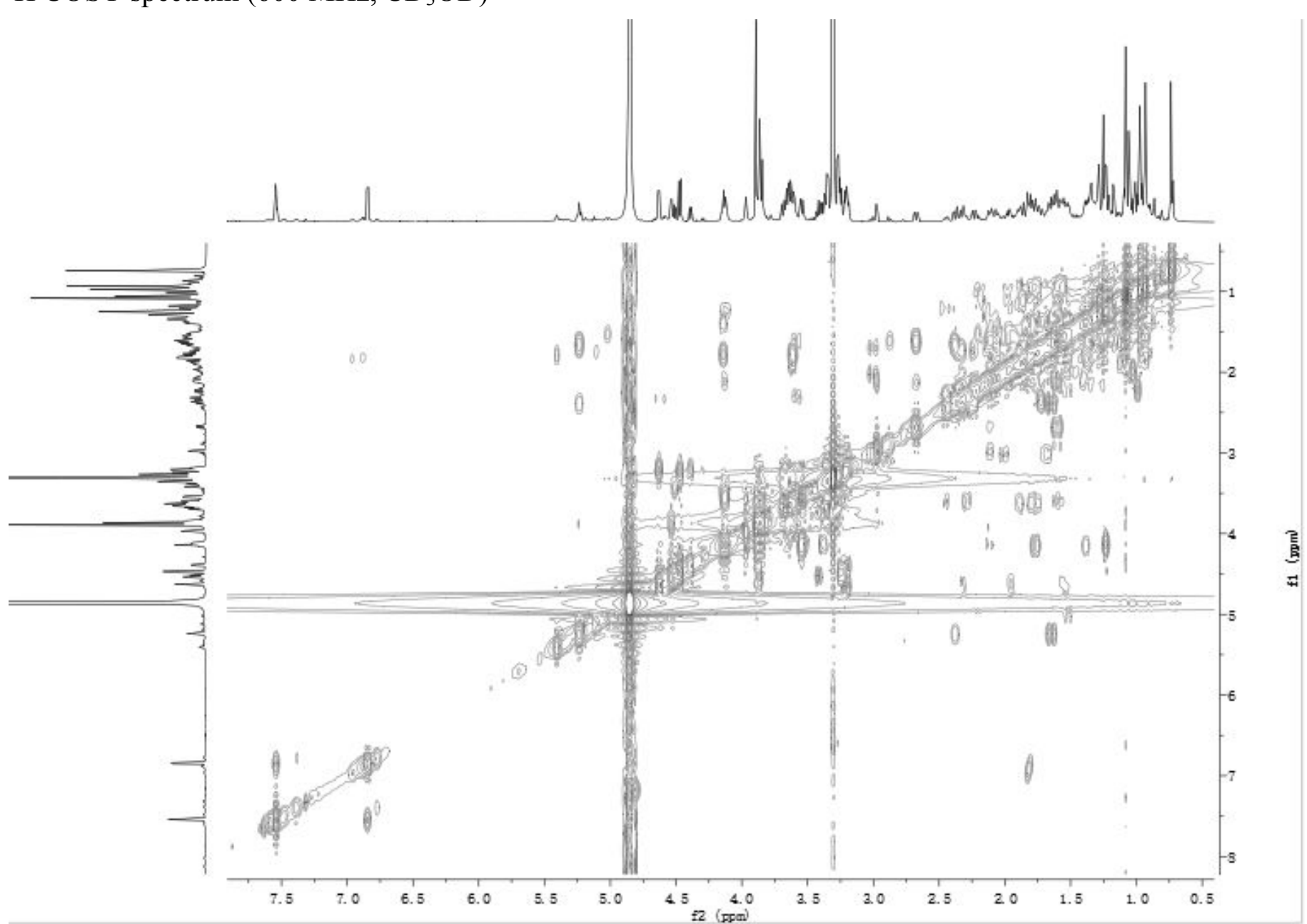


Compound 1: $\mathrm{HMBC}$ spectrum (600 MHz, $\left.\mathrm{CD}_{3} \mathrm{OD}\right)$

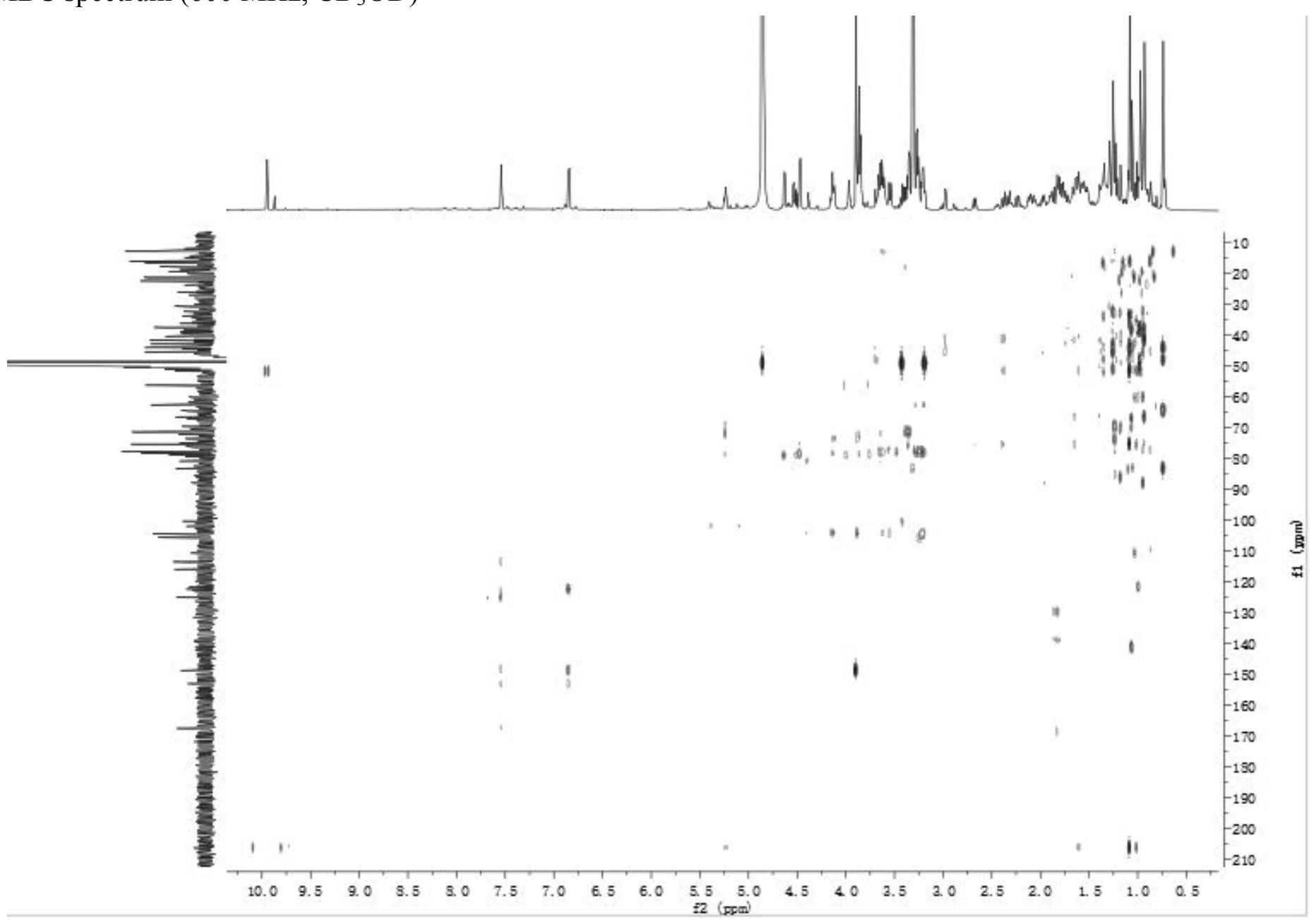


Compound 1: NOESY spectrum (600 MHz, $\left.\mathrm{CD}_{3} \mathrm{OD}\right)$

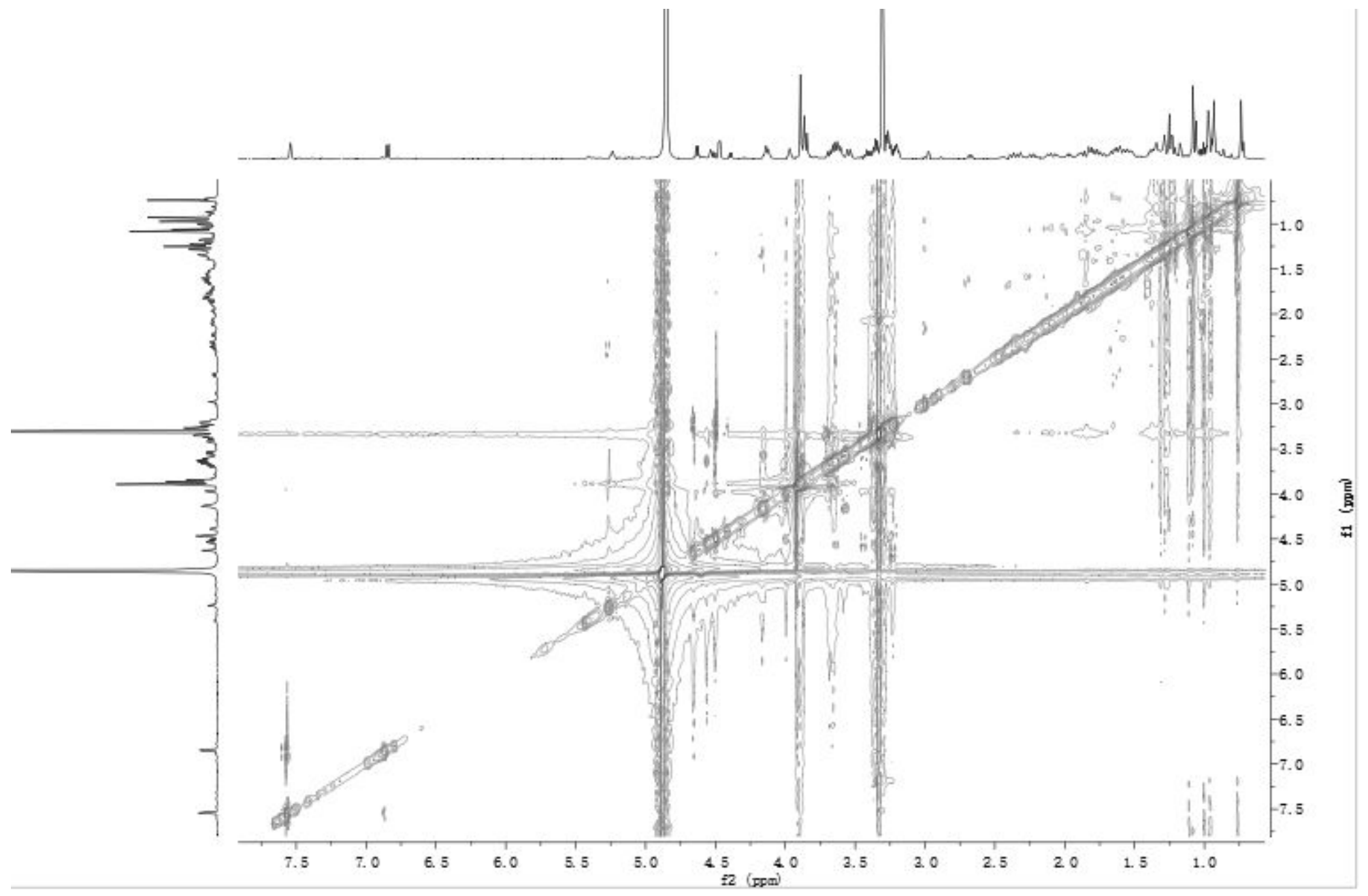


Compound 2: ${ }^{1} \mathrm{H}$ NMR spectrum $\left(600 \mathrm{MHz}, \mathrm{CD}_{3} \mathrm{OD}\right)$

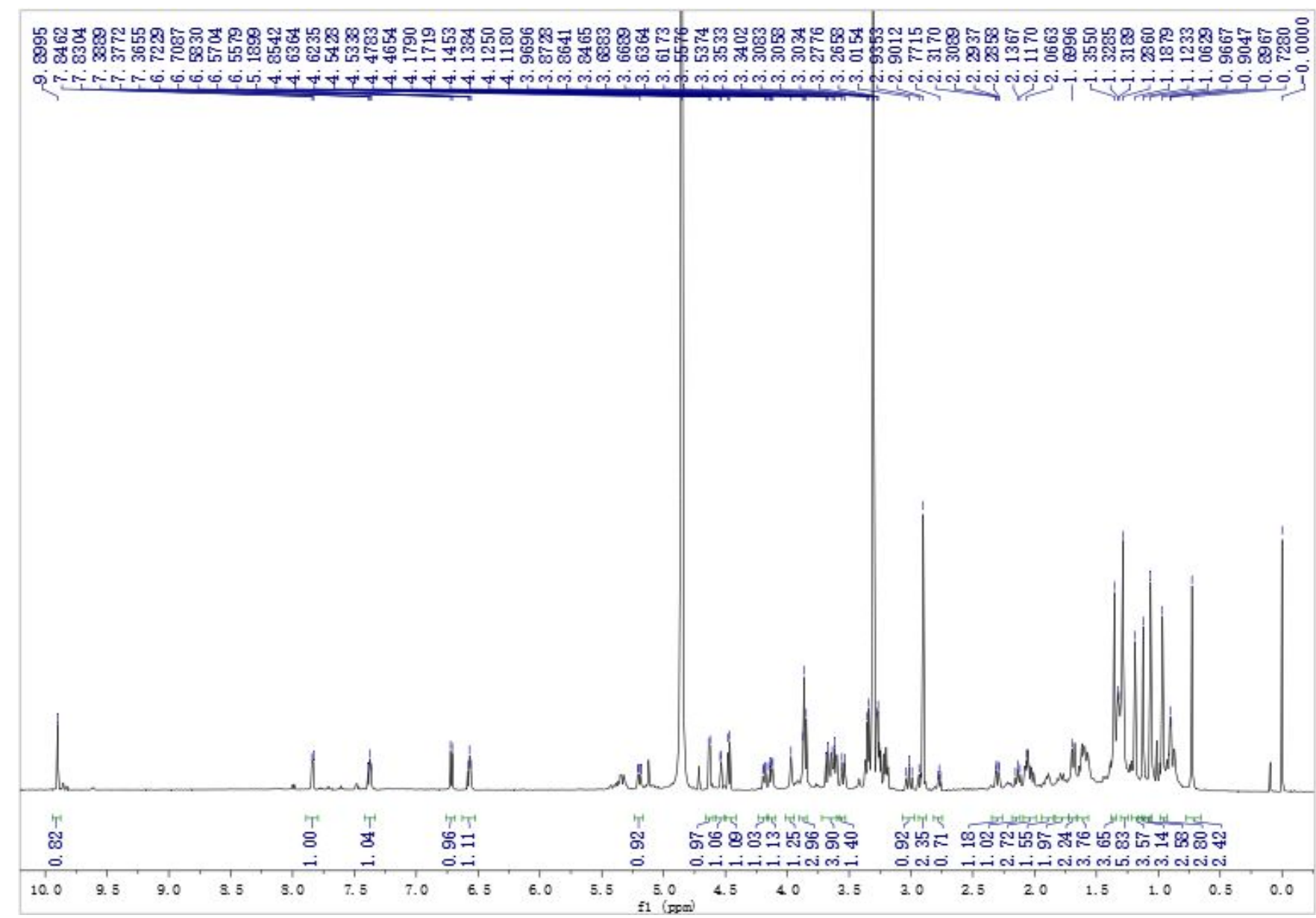


Compound 2: ${ }^{1} \mathrm{H}$ NMR spectrum (600 MHz, $\mathrm{CD}_{3} \mathrm{OD}$ )-expansion

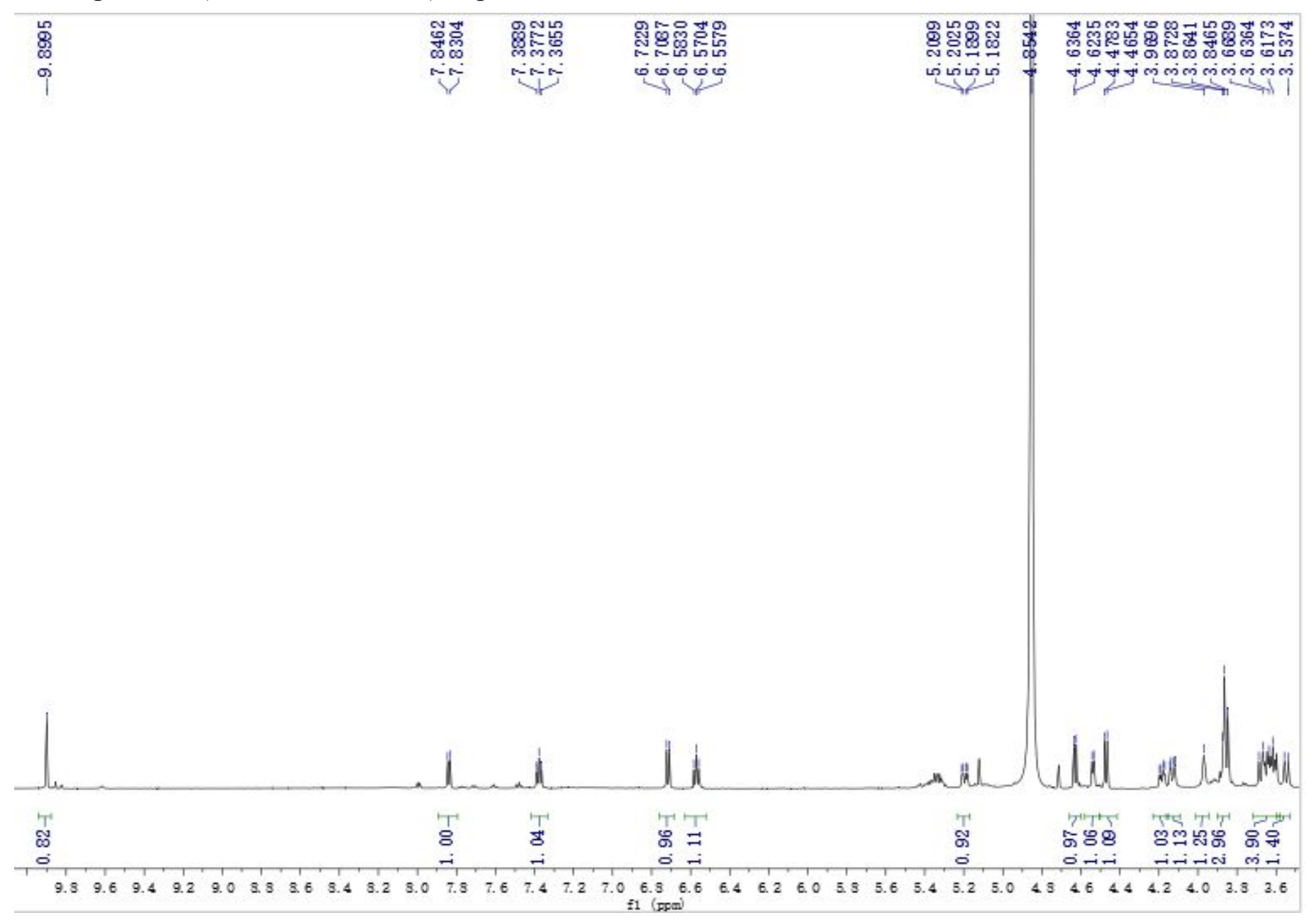


Compound 2: ${ }^{1} \mathrm{H}$ NMR spectrum (600 MHz, $\mathrm{CD}_{3} \mathrm{OD}$ )-expansion

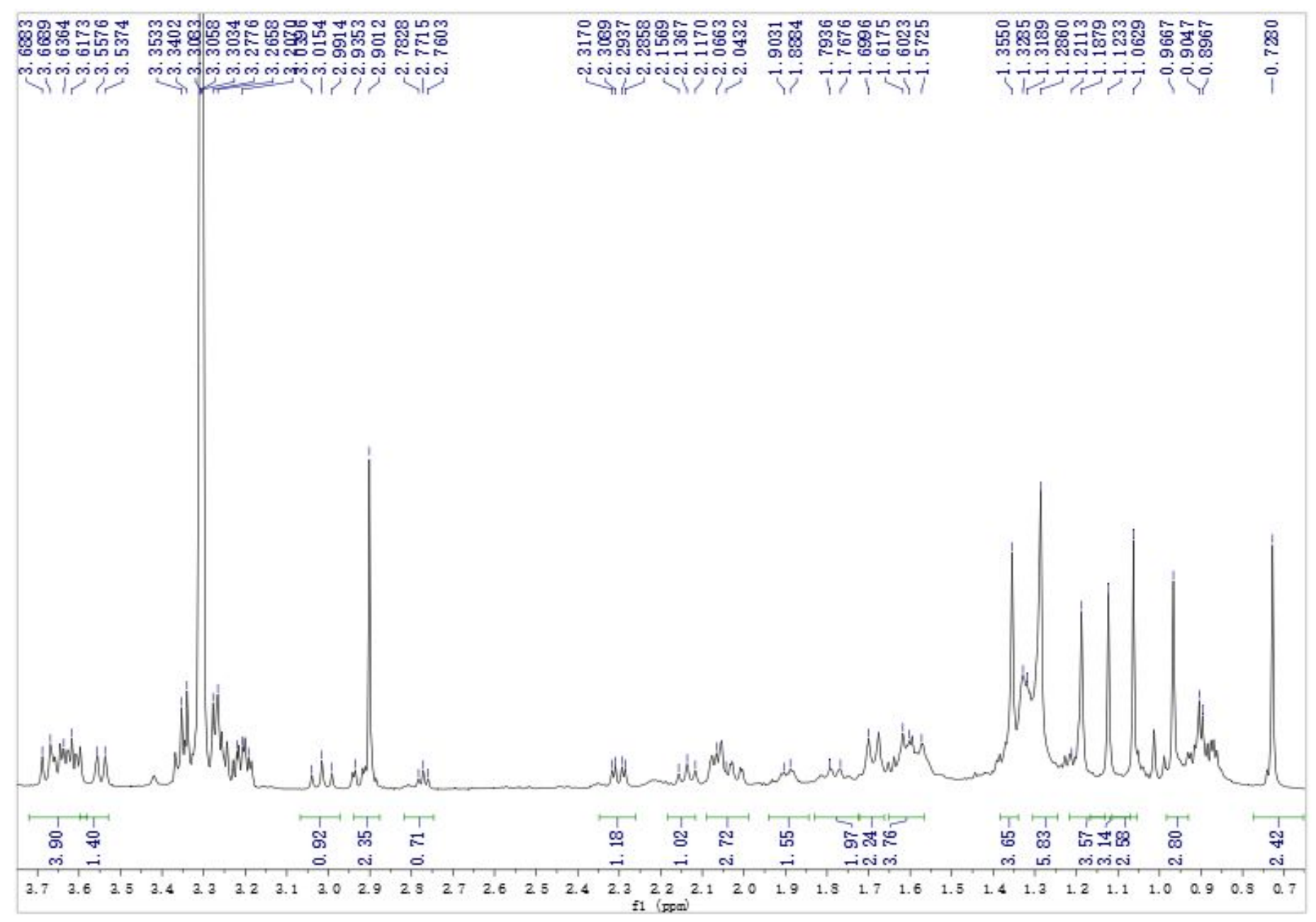


Compound 2: ${ }^{13} \mathrm{C}$ NMR spectrum $\left(600 \mathrm{MHz}, \mathrm{CD}_{3} \mathrm{OD}\right)$

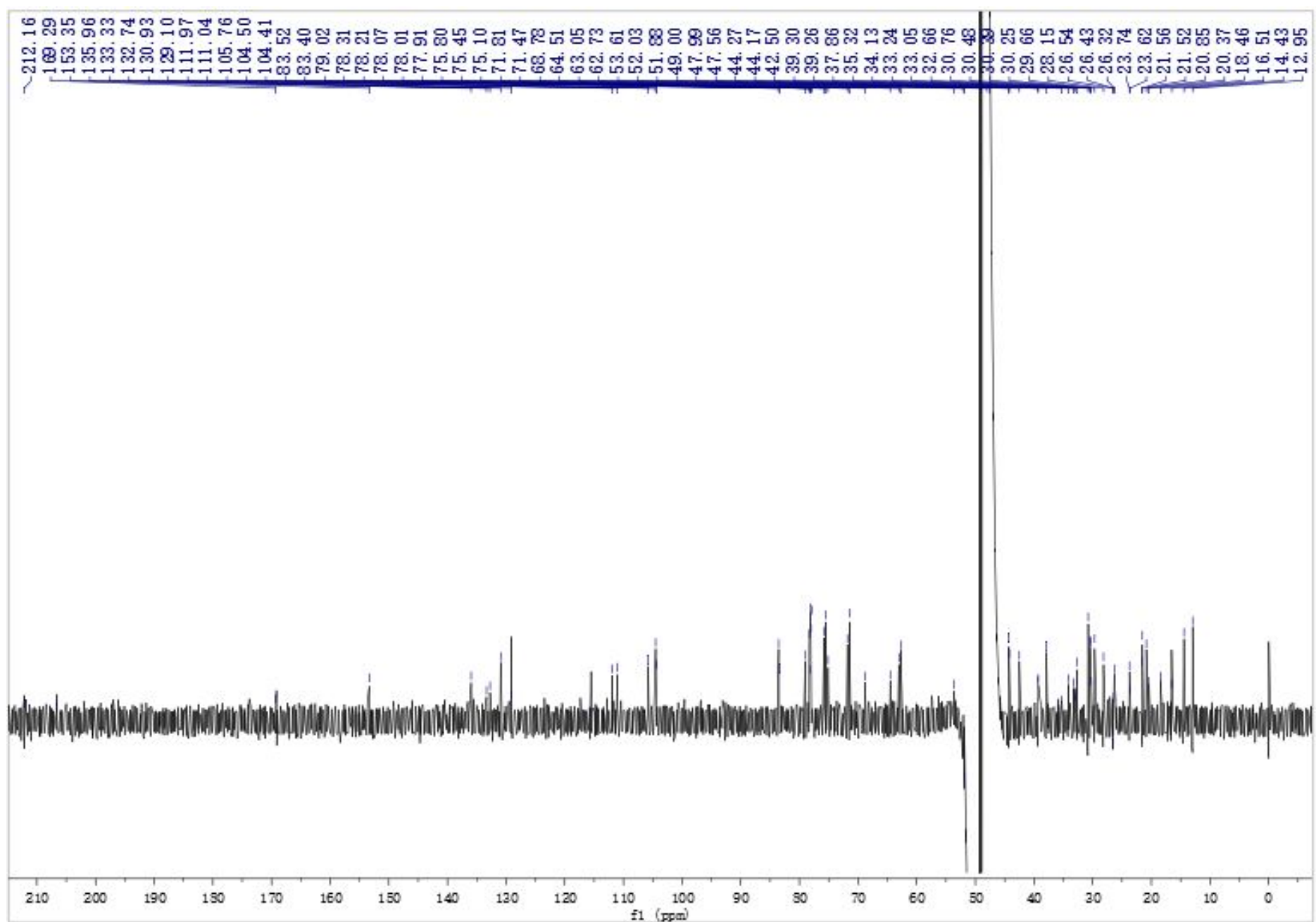


Compound 2: ${ }^{13} \mathrm{C}$ NMR spectrum (600 MHz, $\mathrm{CD}_{3} \mathrm{OD}$ )-expansion

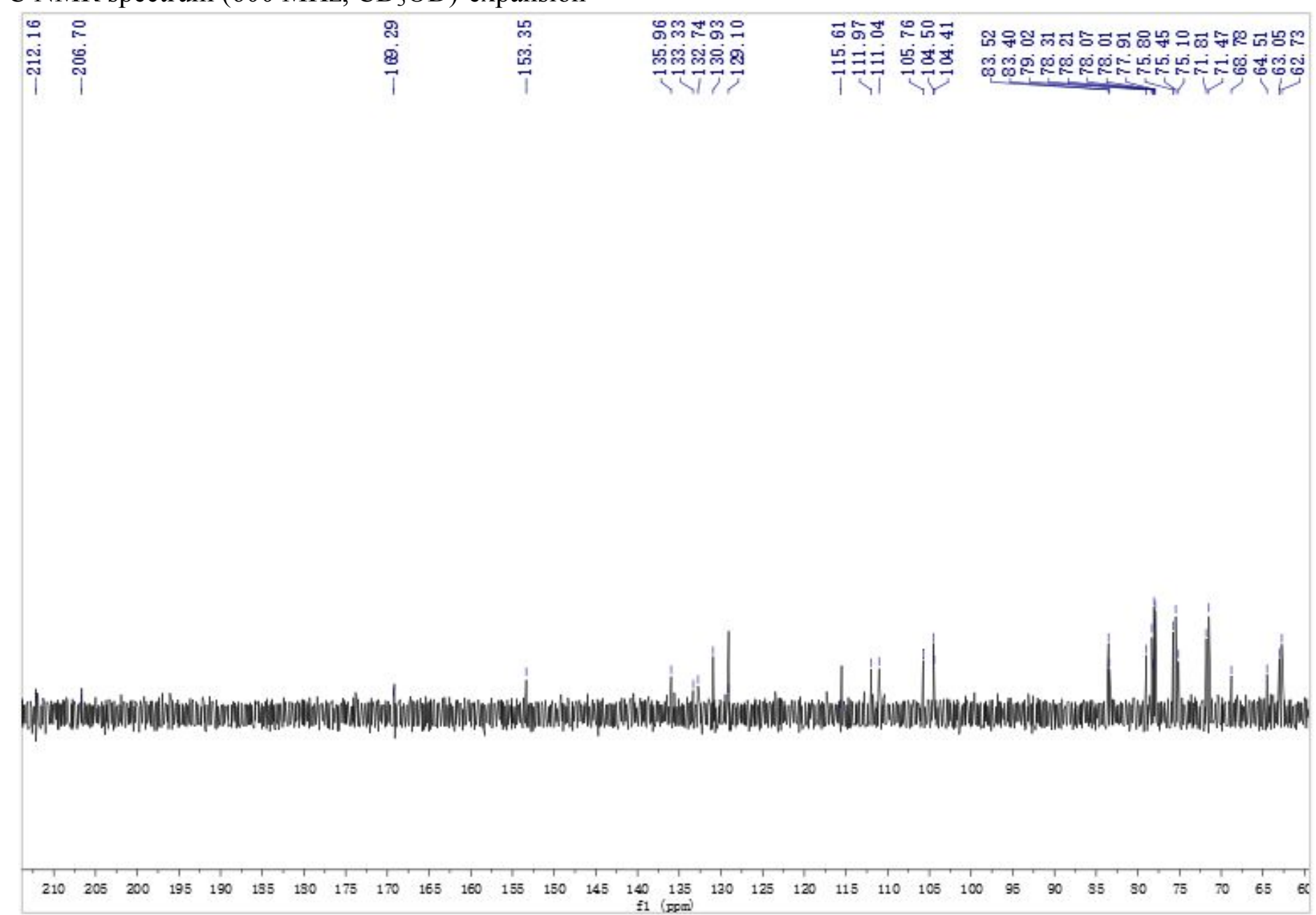


Compound 2: ${ }^{13} \mathrm{C}$ NMR spectrum (600 MHz, $\left.\mathrm{CD}_{3} \mathrm{OD}\right)$-expansion

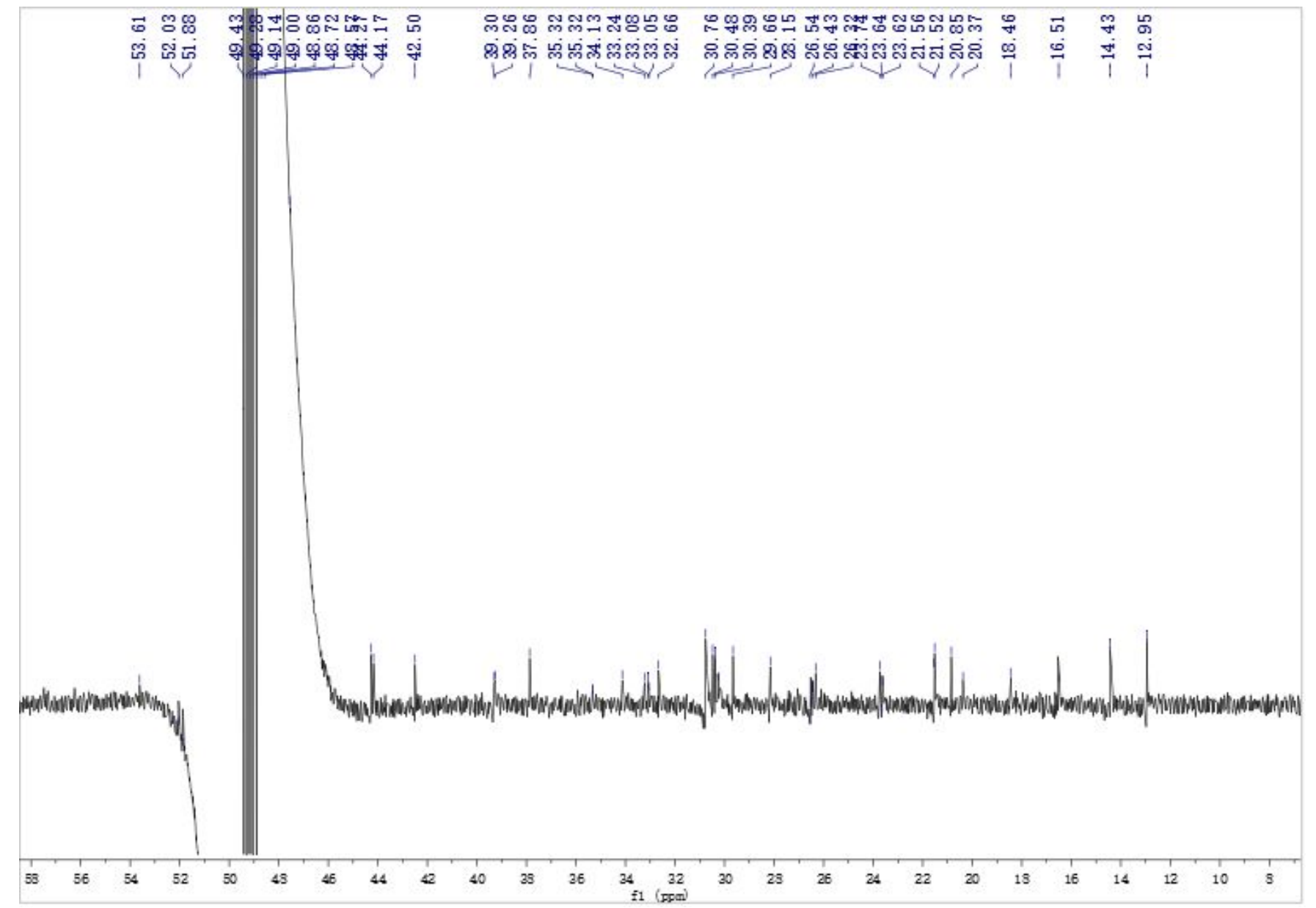




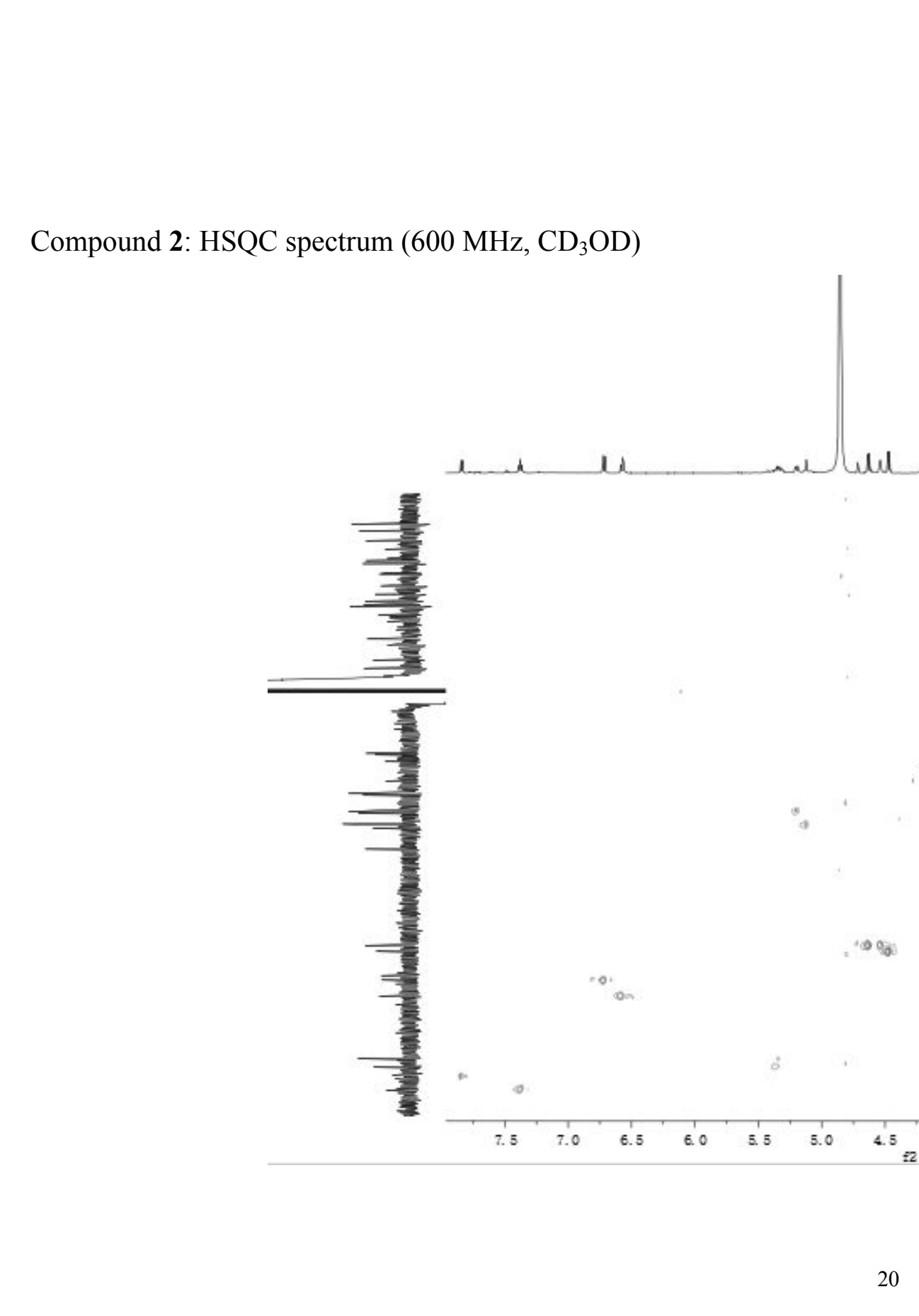


Compound 2: ${ }^{1} \mathrm{H}-{ }^{1} \mathrm{H}$ COSY spectrum $\left(600 \mathrm{MHz}, \mathrm{CD}_{3} \mathrm{OD}\right)$

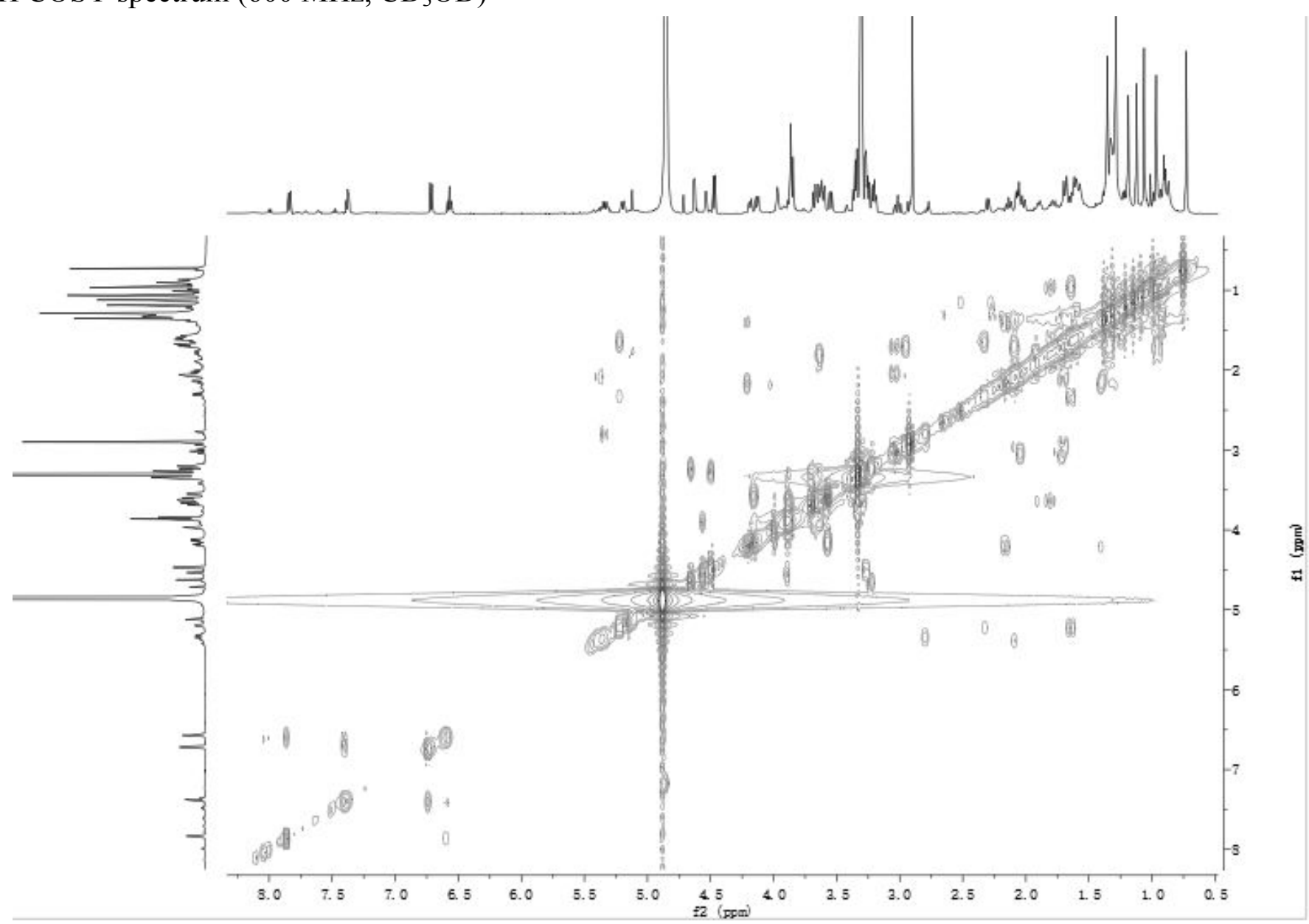


Compound 2: $\mathrm{HMBC}$ spectrum (600 MHz, $\left.\mathrm{CD}_{3} \mathrm{OD}\right)$

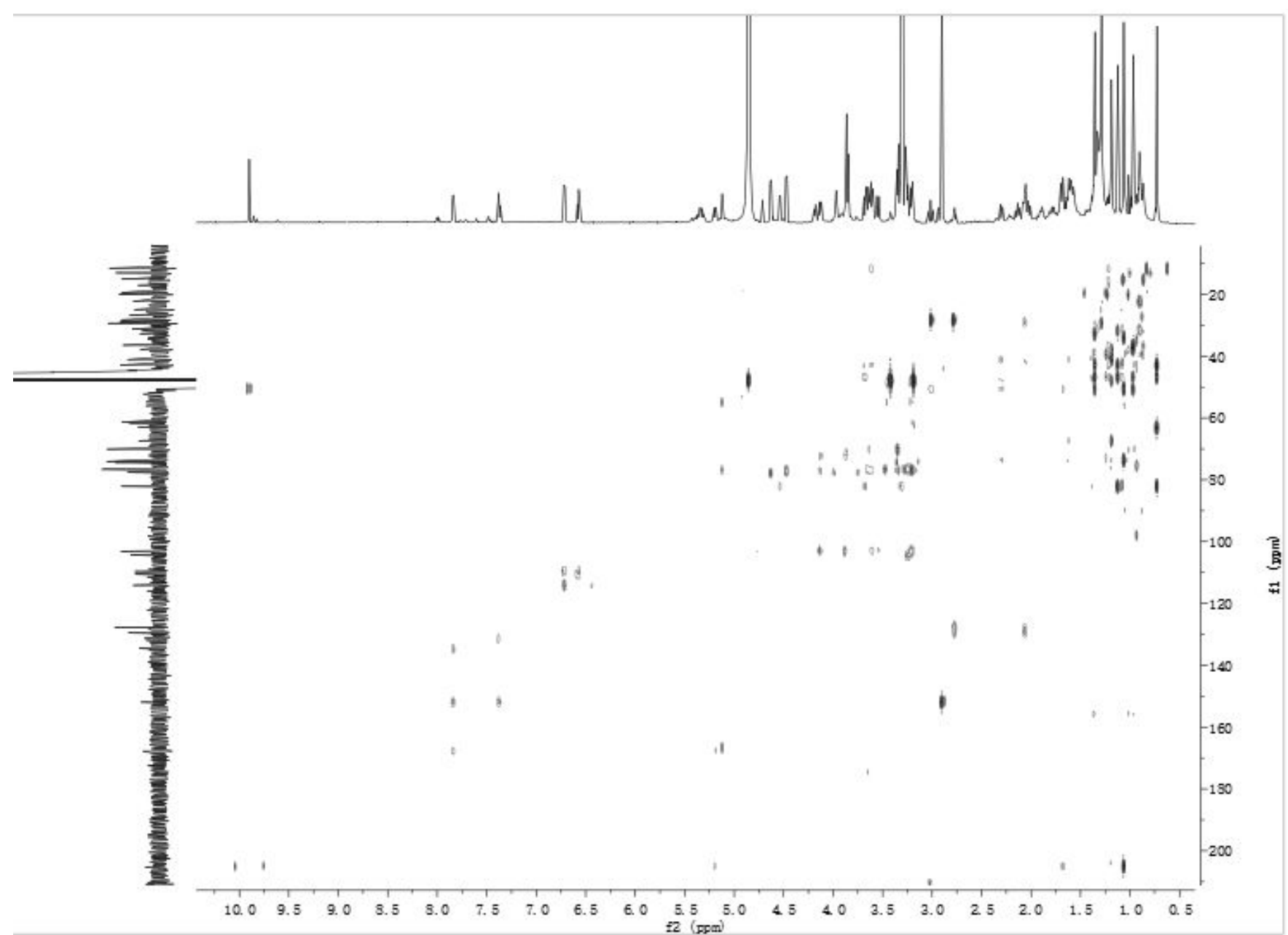


Compound 2: NOESY spectrum (600 MHz, $\mathrm{CD}_{3} \mathrm{OD}$ )

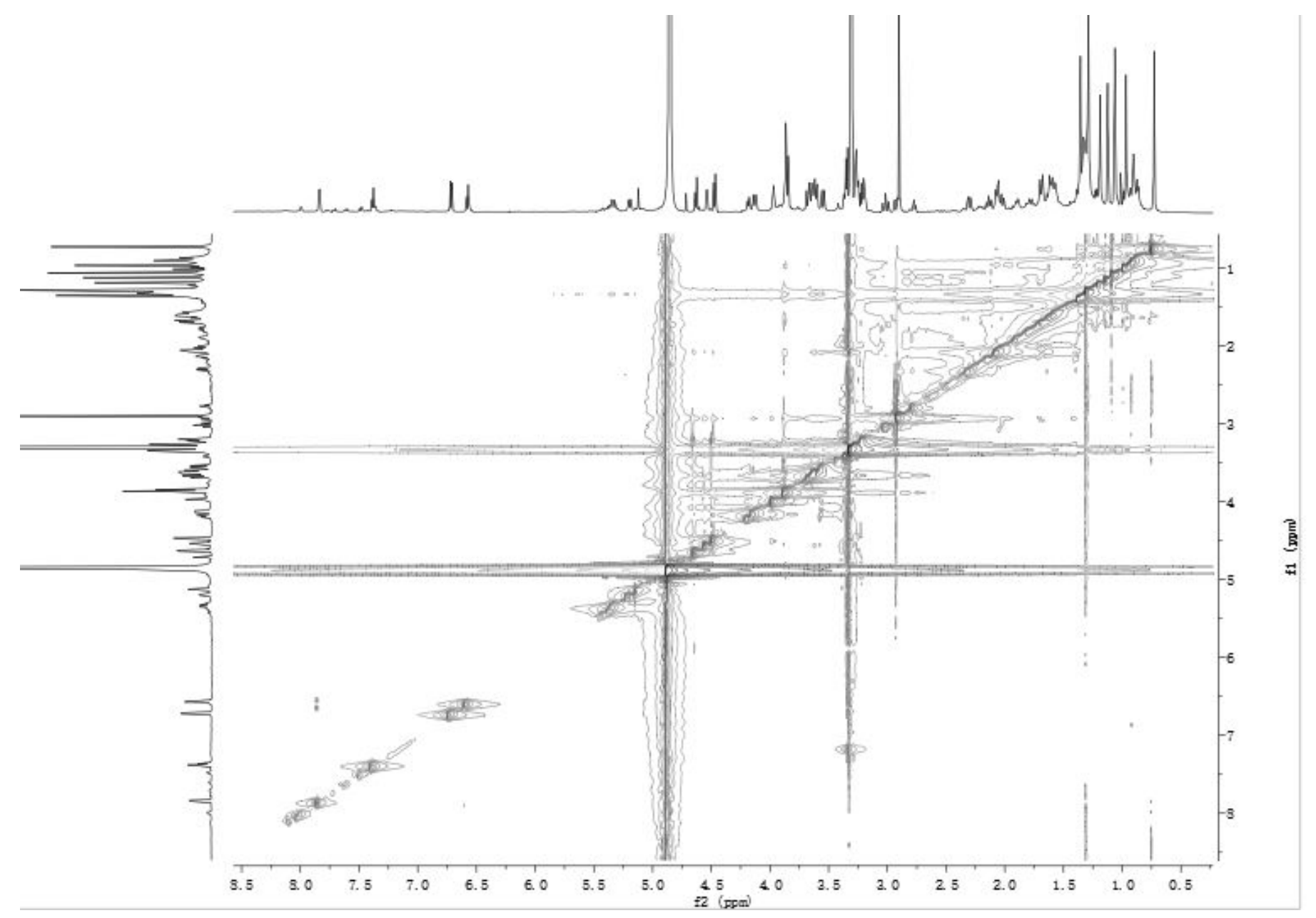

23 
Compound 3: ${ }^{1} \mathrm{H}$ NMR spectrum (600 MHz, $\left.\mathrm{CD}_{3} \mathrm{OD}\right)$

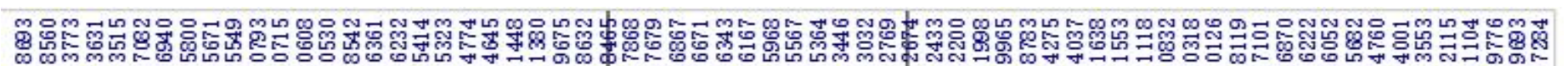

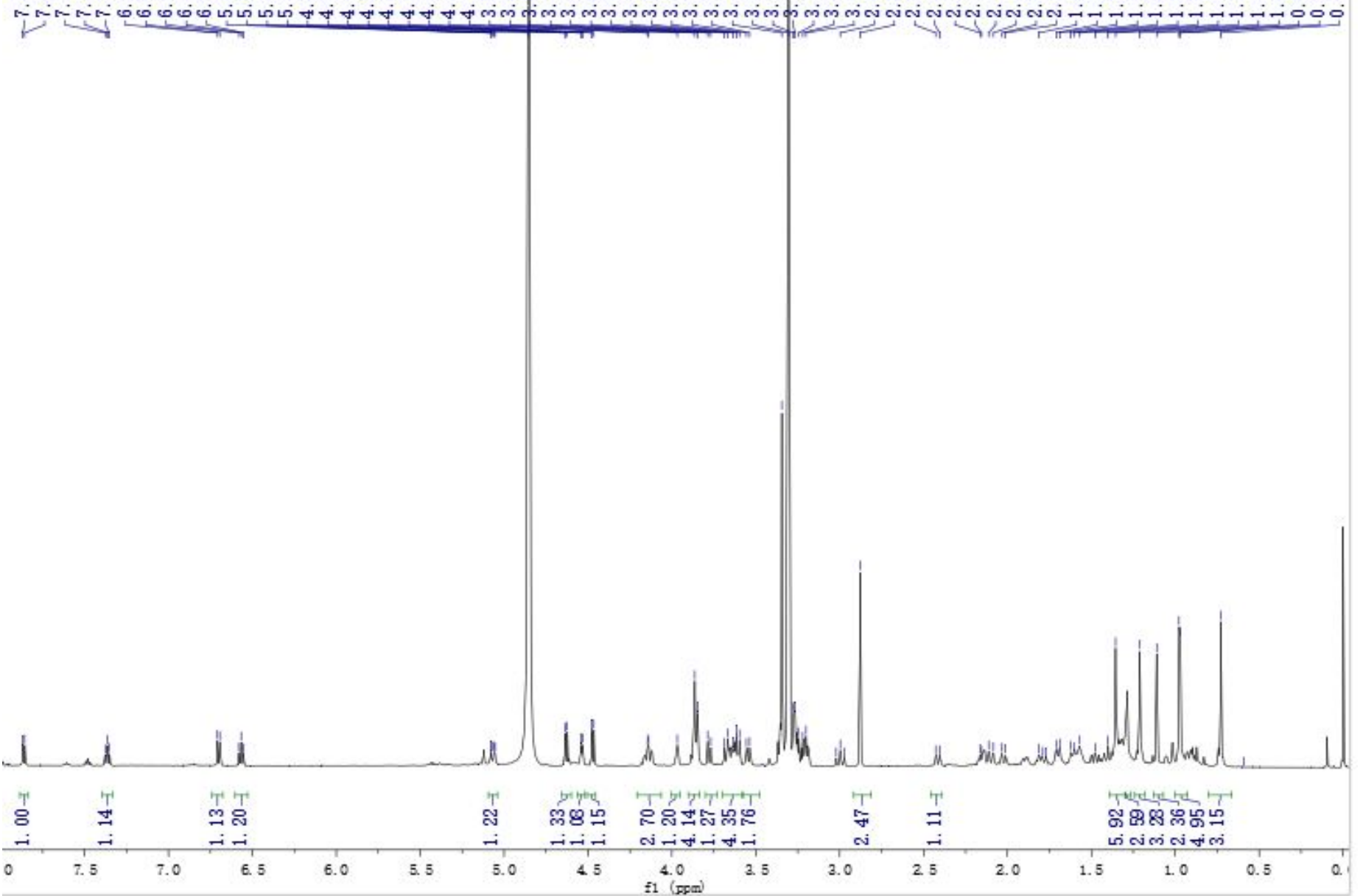


Compound 3: ${ }^{13} \mathrm{C}$ NMR spectrum $\left(600 \mathrm{MHz}, \mathrm{CD}_{3} \mathrm{OD}\right)$

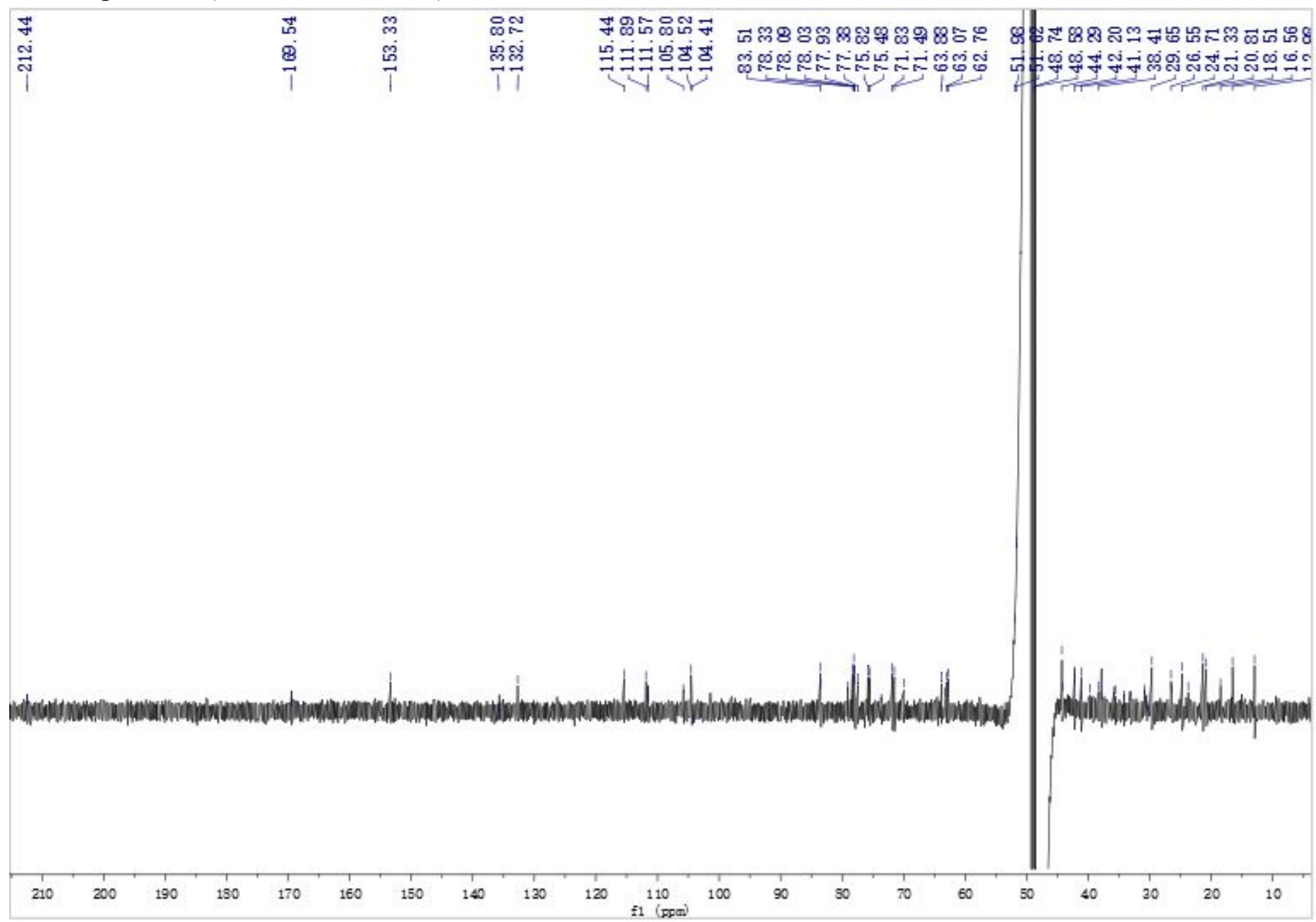


Compound 3: HSQC spectrum (600 MHz, $\left.\mathrm{CD}_{3} \mathrm{OD}\right)$

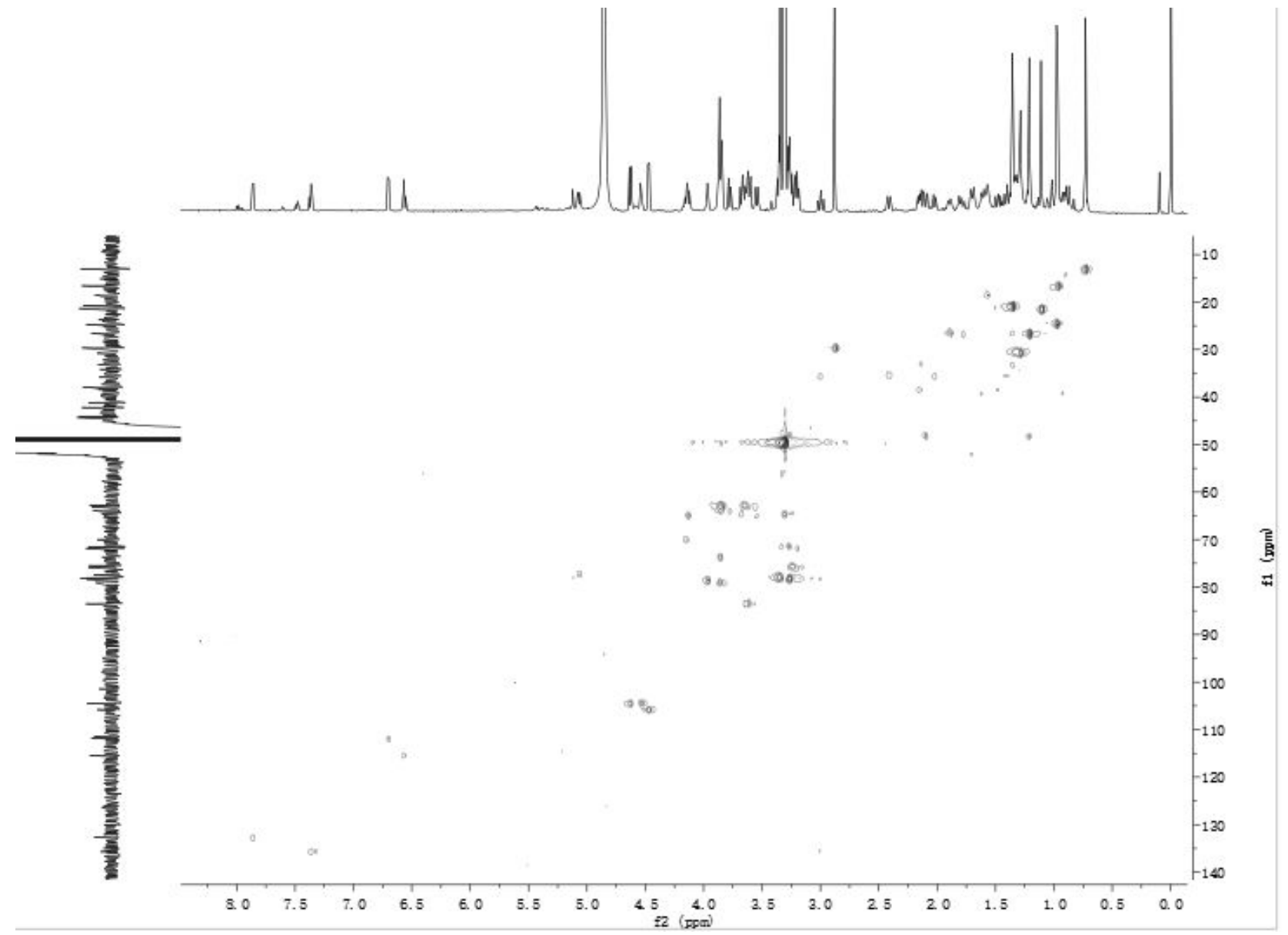


Compound 3: $\mathrm{HMBC}$ spectrum $\left(600 \mathrm{MHz}, \mathrm{CD}_{3} \mathrm{OD}\right)$

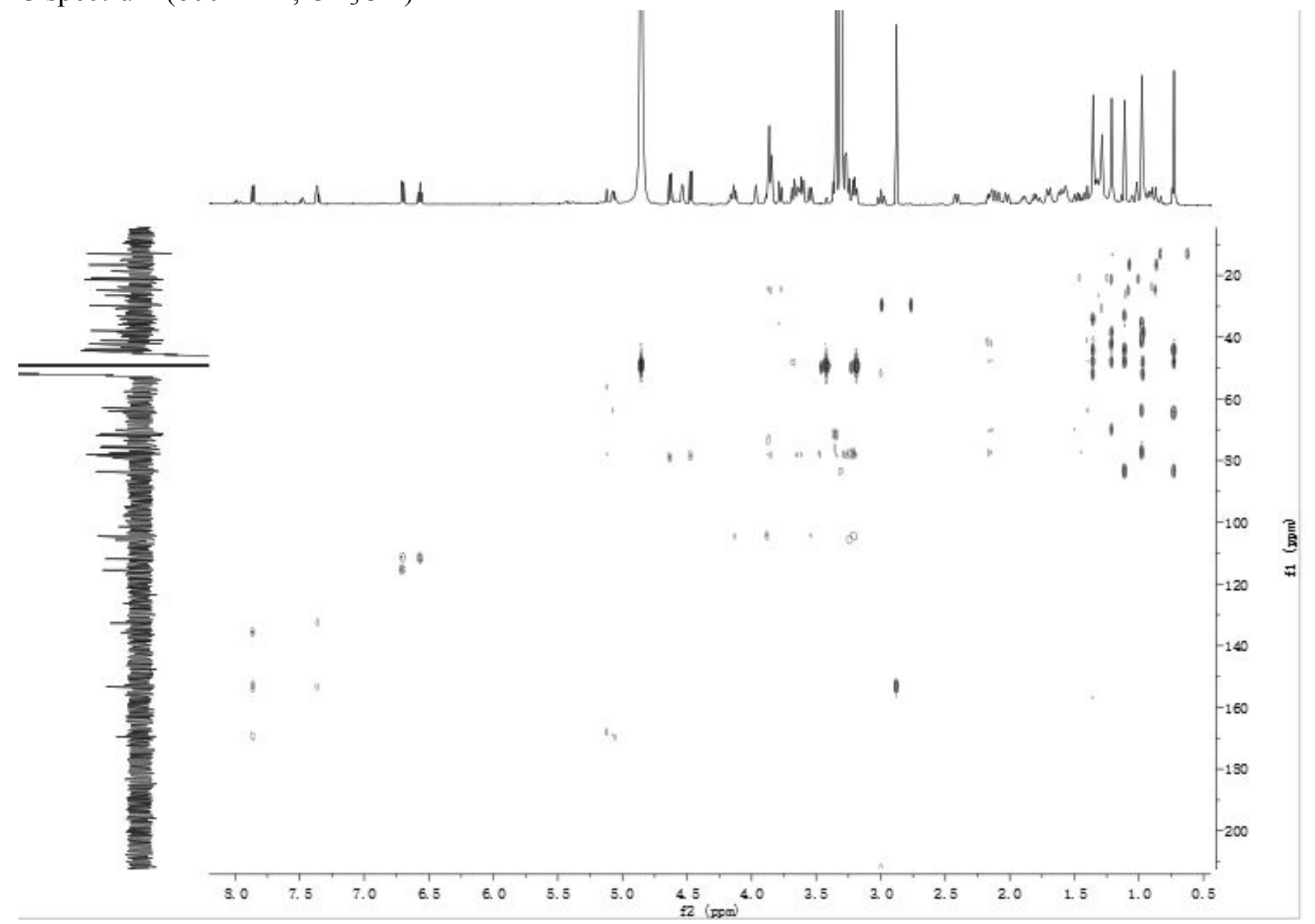


Compound 3: NOESY spectrum (600 MHz, $\left.\mathrm{CD}_{3} \mathrm{OD}\right)$

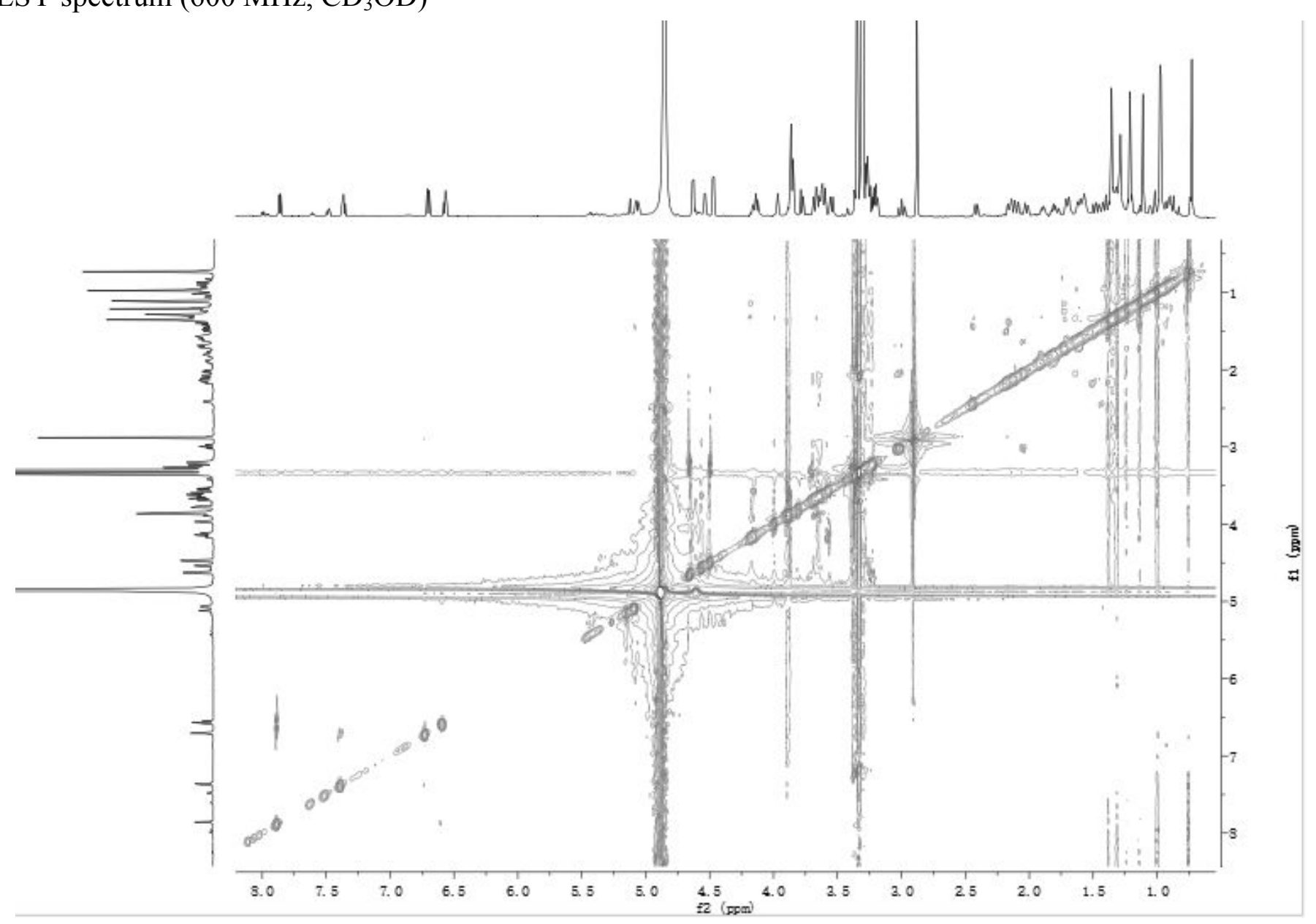


Compound 4: ${ }^{1} \mathrm{H}$ NMR spectrum $\left(600 \mathrm{MHz}, \mathrm{CD}_{3} \mathrm{OD}\right)$

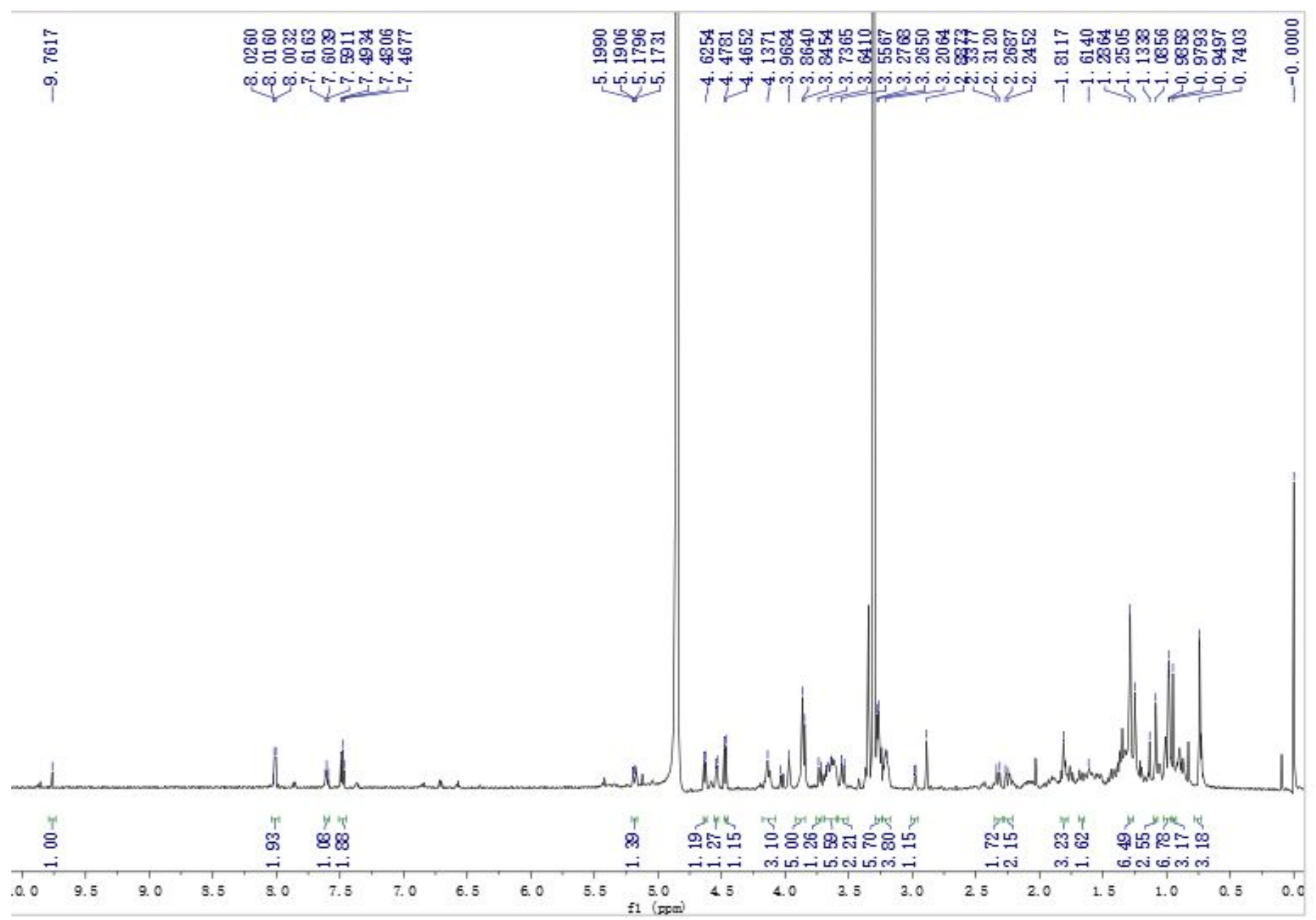


Compound 4: ${ }^{13} \mathrm{C}$ NMR spectrum $\left(600 \mathrm{MHz}, \mathrm{CD}_{3} \mathrm{OD}\right)$

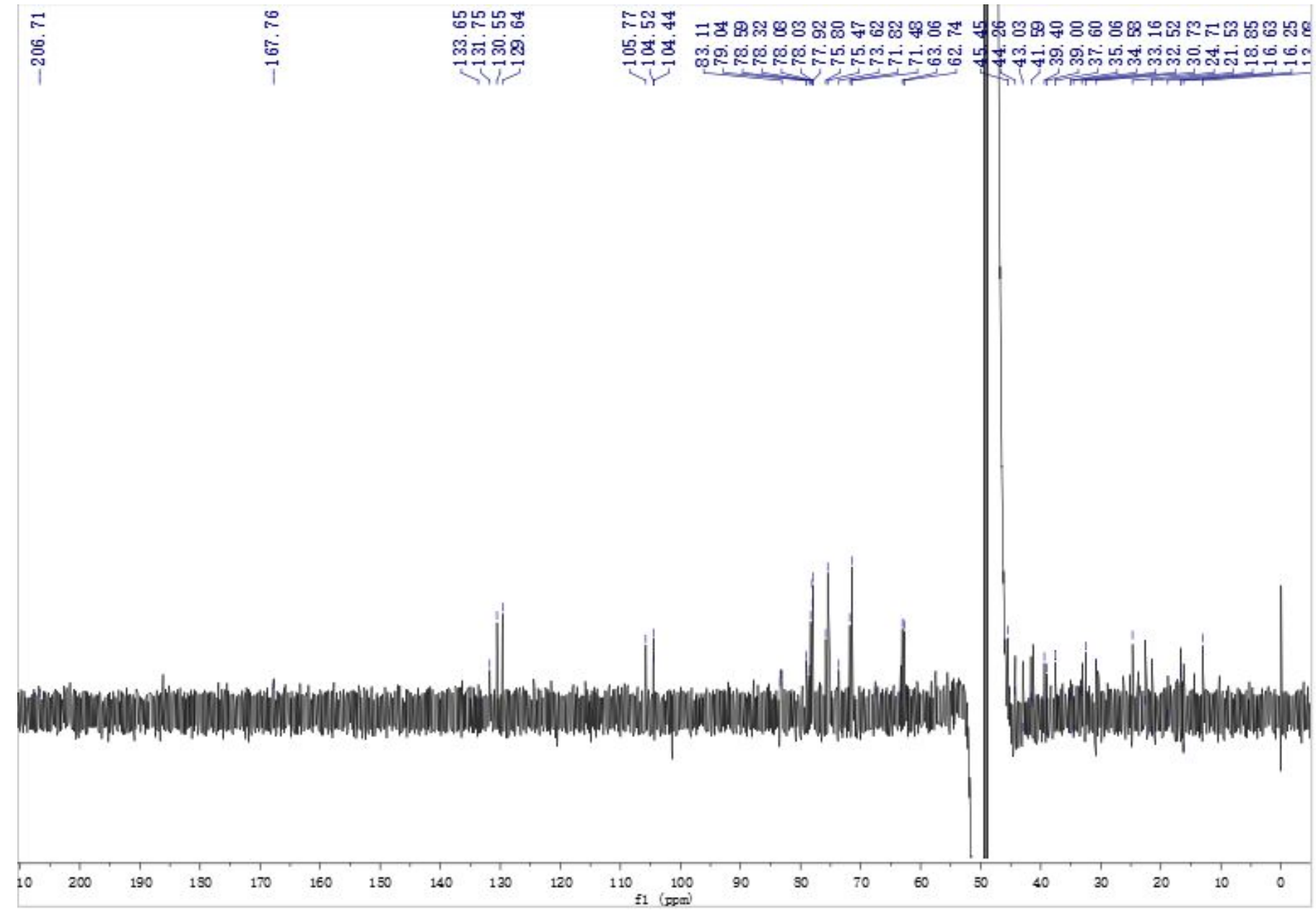


Compound 4: $\mathrm{HMBC}$ spectrum (600 MHz, $\left.\mathrm{CD}_{3} \mathrm{OD}\right)$

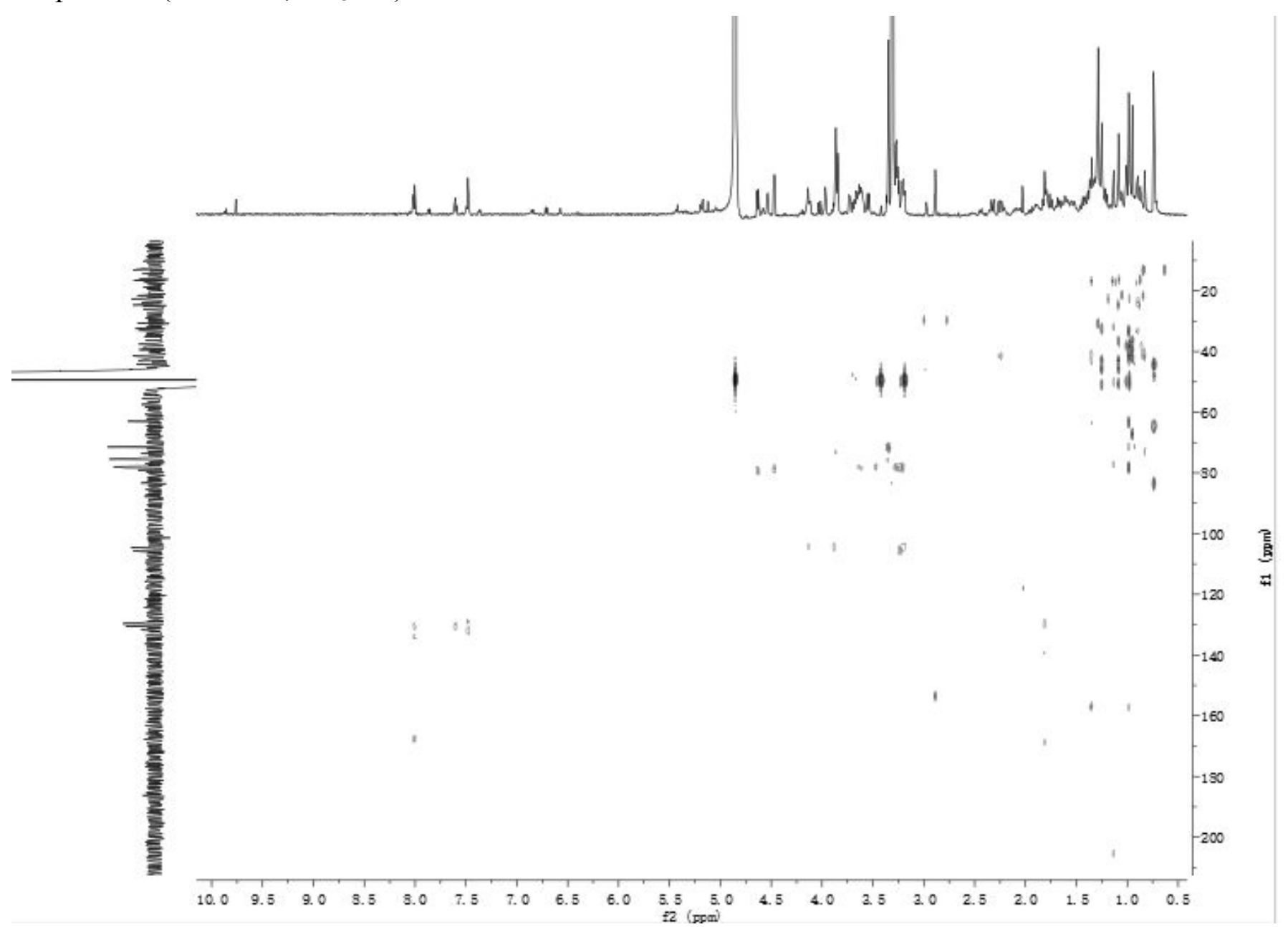


Compound 4: NOESY spectrum (600 MHz, $\left.\mathrm{CD}_{3} \mathrm{OD}\right)$

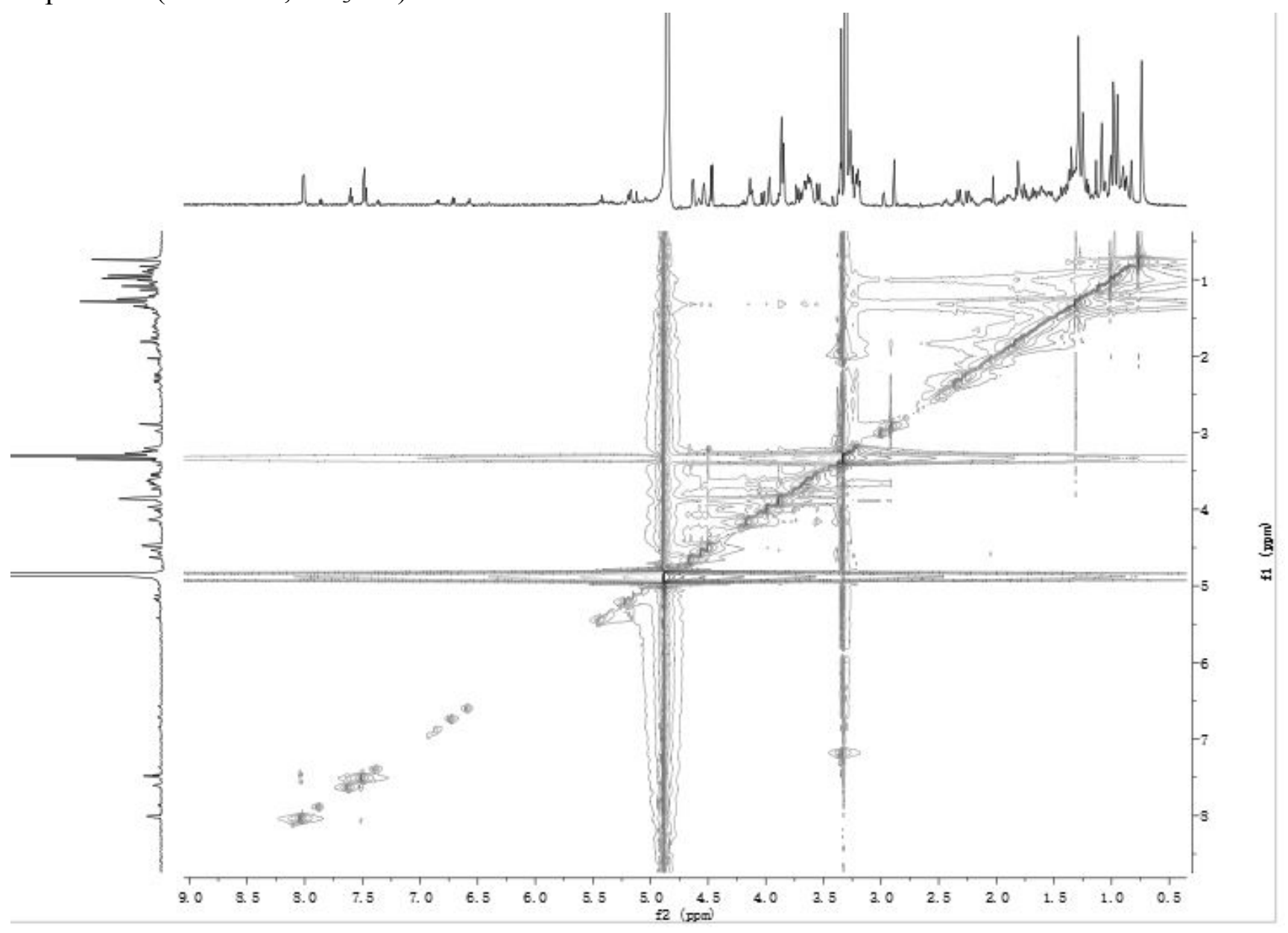

32 
Compound 5: ${ }^{1} \mathrm{H}$ NMR spectrum (600 MHz, $\left.\mathrm{CD}_{3} \mathrm{OD}\right)$

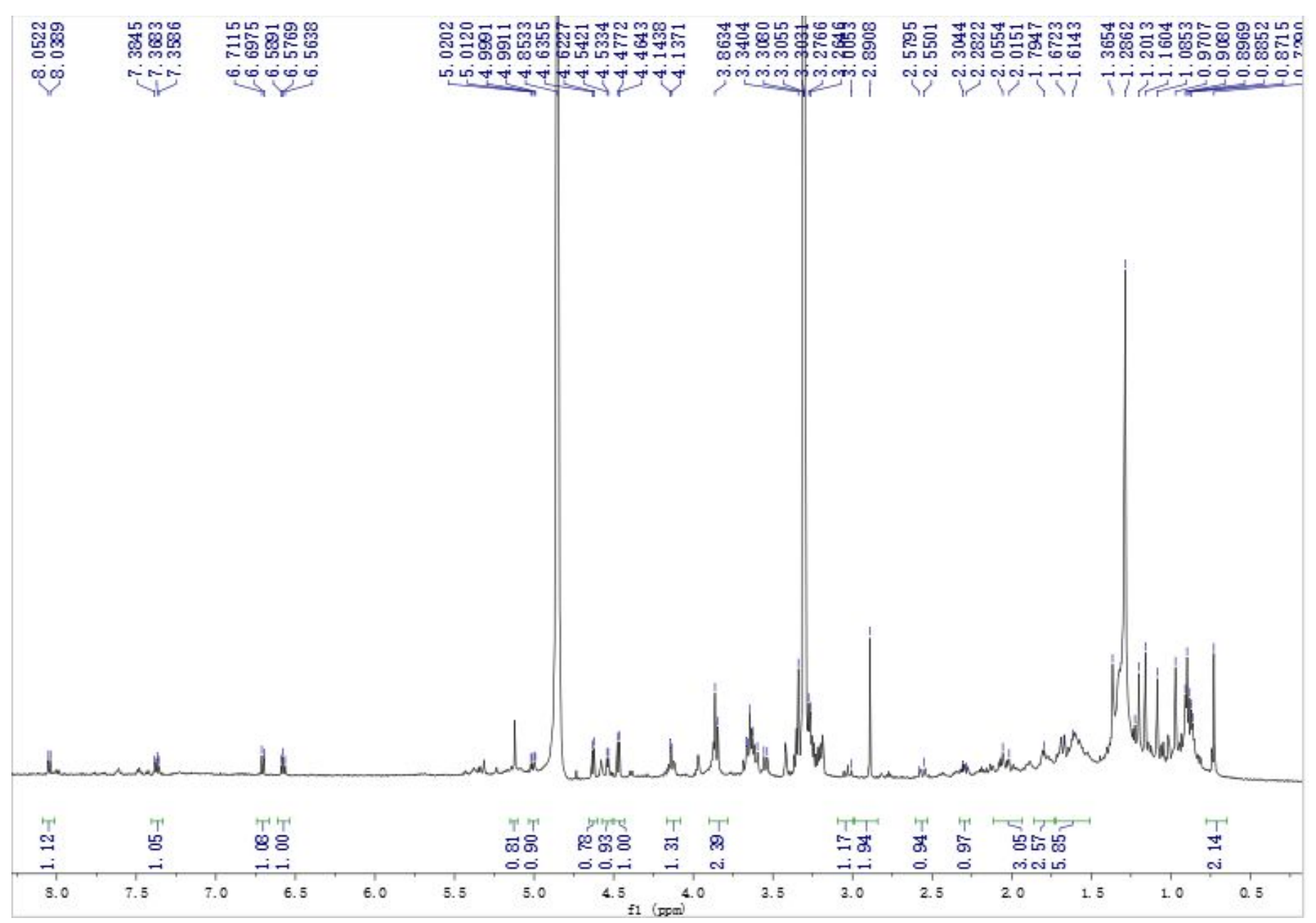


Compound 6: ${ }^{1} \mathrm{H}$ NMR spectrum (600 MHz, $\left.\mathrm{CD}_{3} \mathrm{OD}\right)$

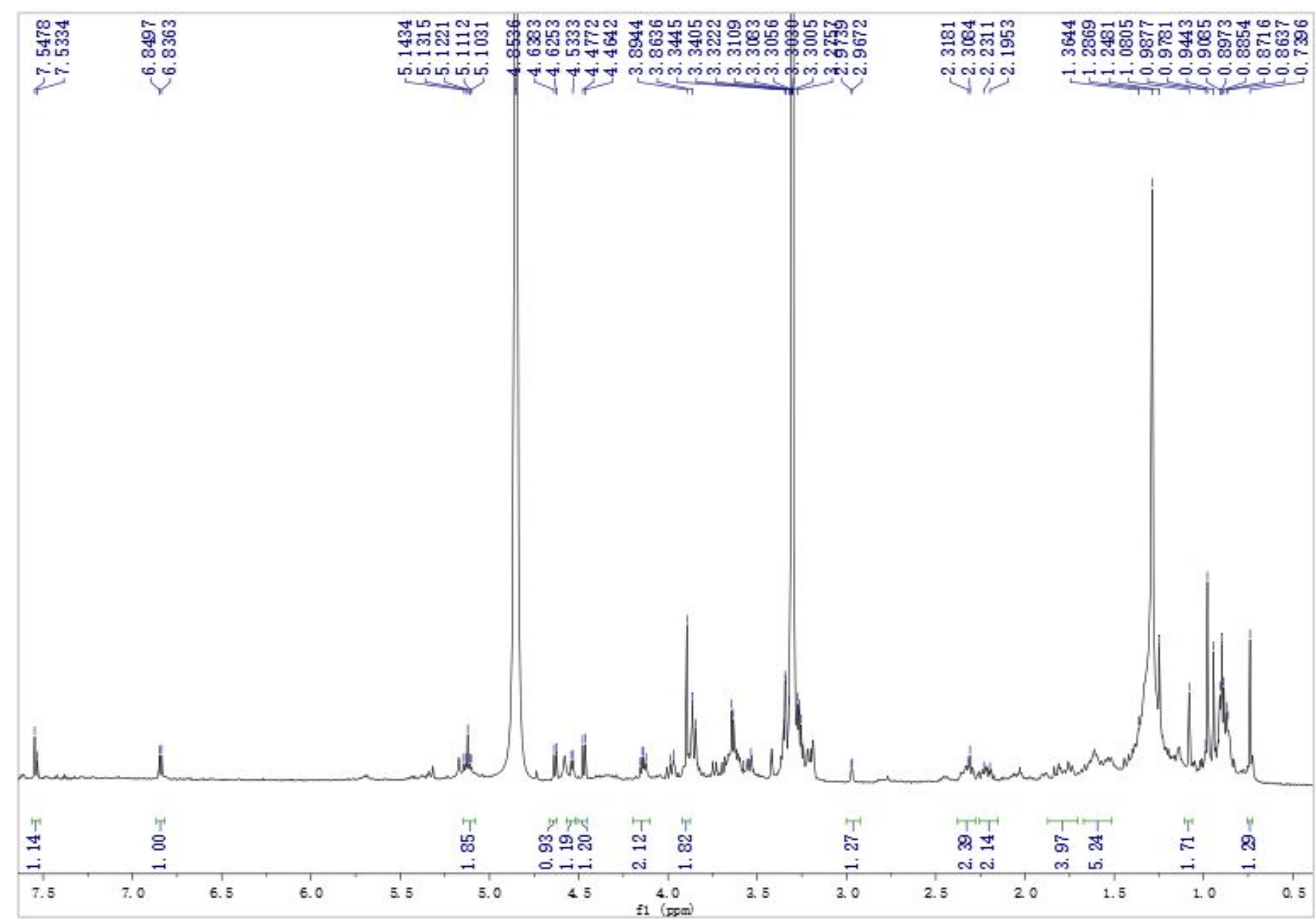

34 
Compound 7: ${ }^{1} \mathrm{H}$ NMR spectrum (600 MHz, $\left.\mathrm{CD}_{3} \mathrm{OD}\right)$

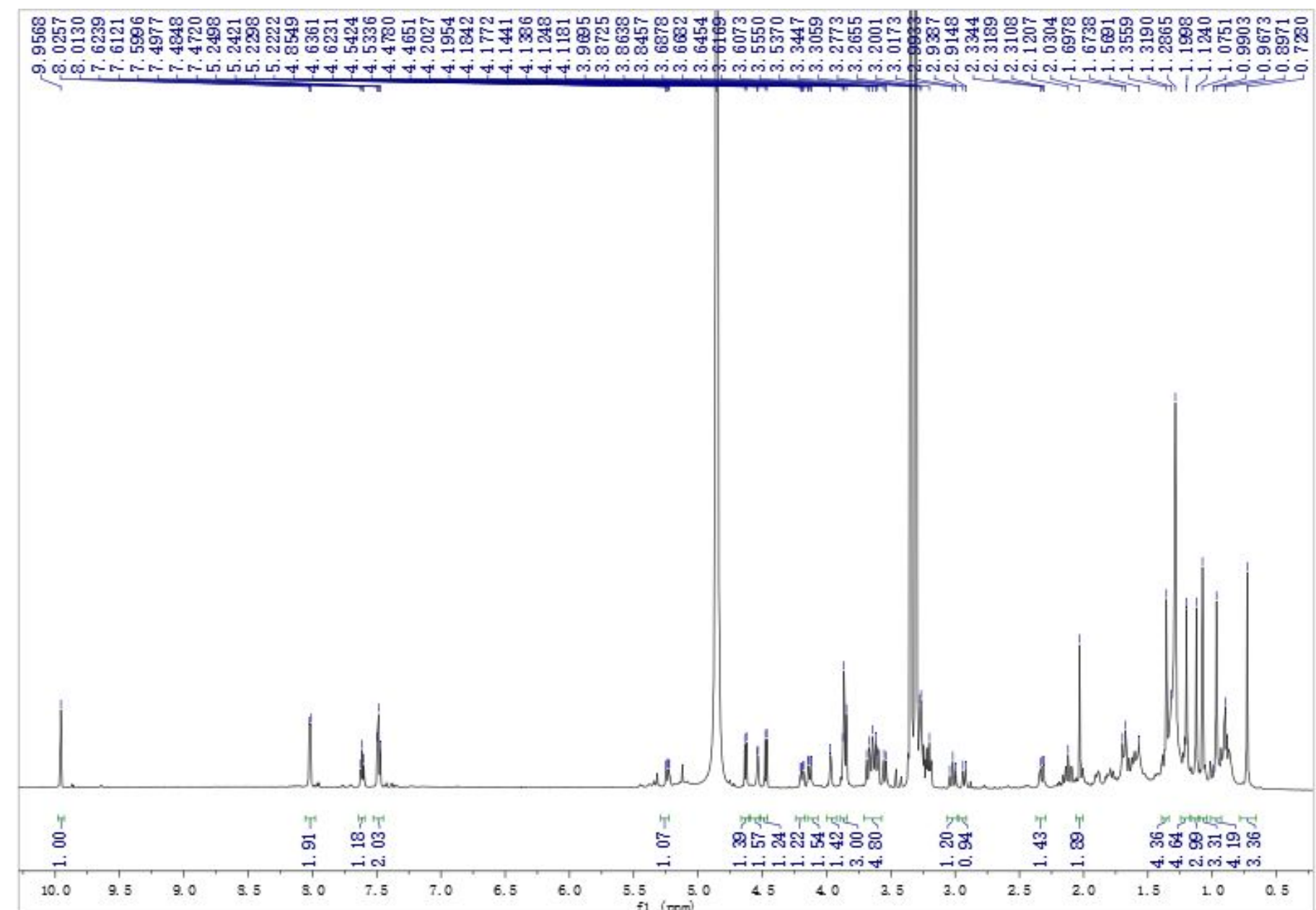


Compound 7: ${ }^{13} \mathrm{C}$ NMR spectrum $\left(600 \mathrm{MHz}, \mathrm{CD}_{3} \mathrm{OD}\right)$

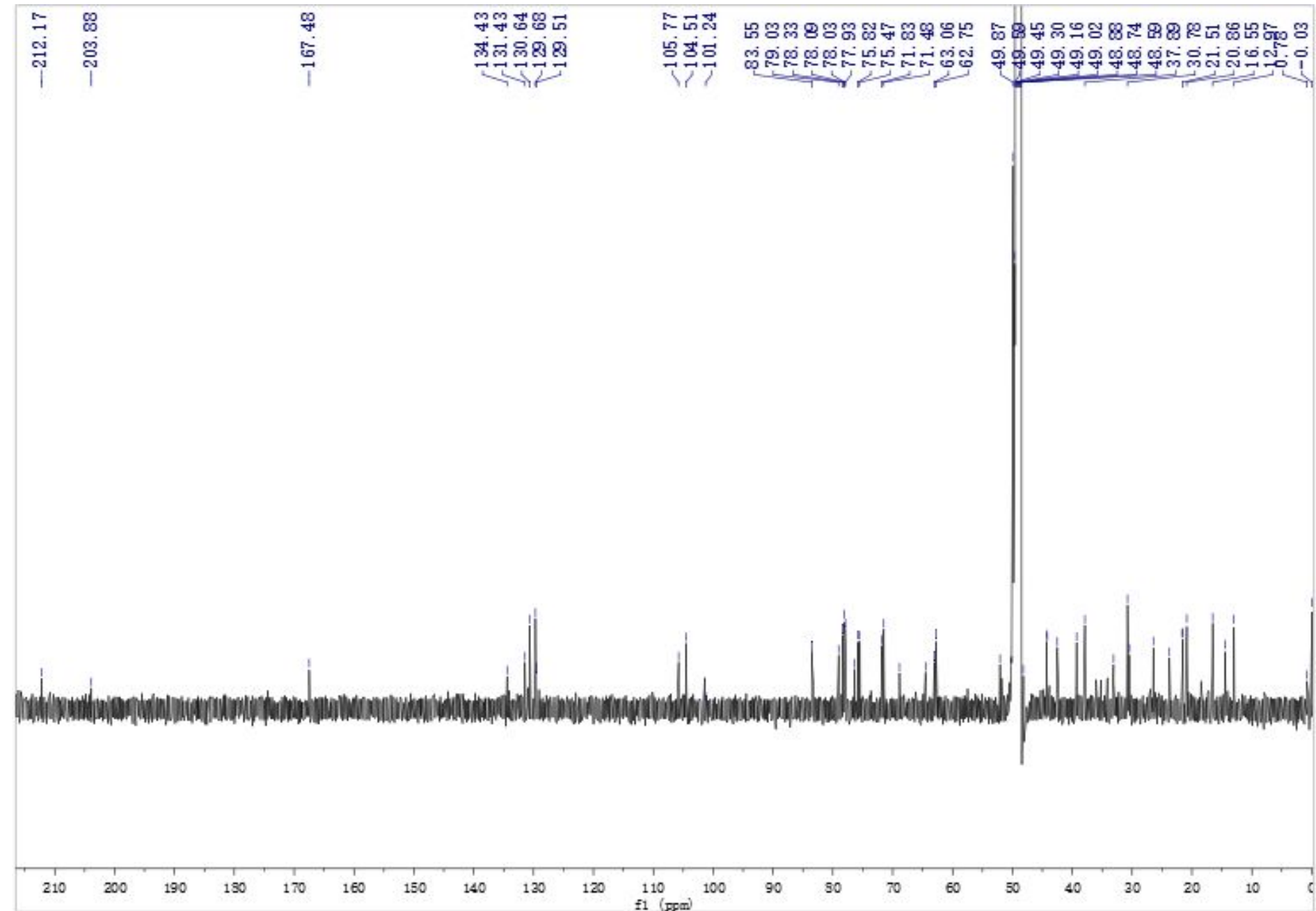


Compound 7: HSQC spectrum (600 MHz, $\left.\mathrm{CD}_{3} \mathrm{OD}\right)$

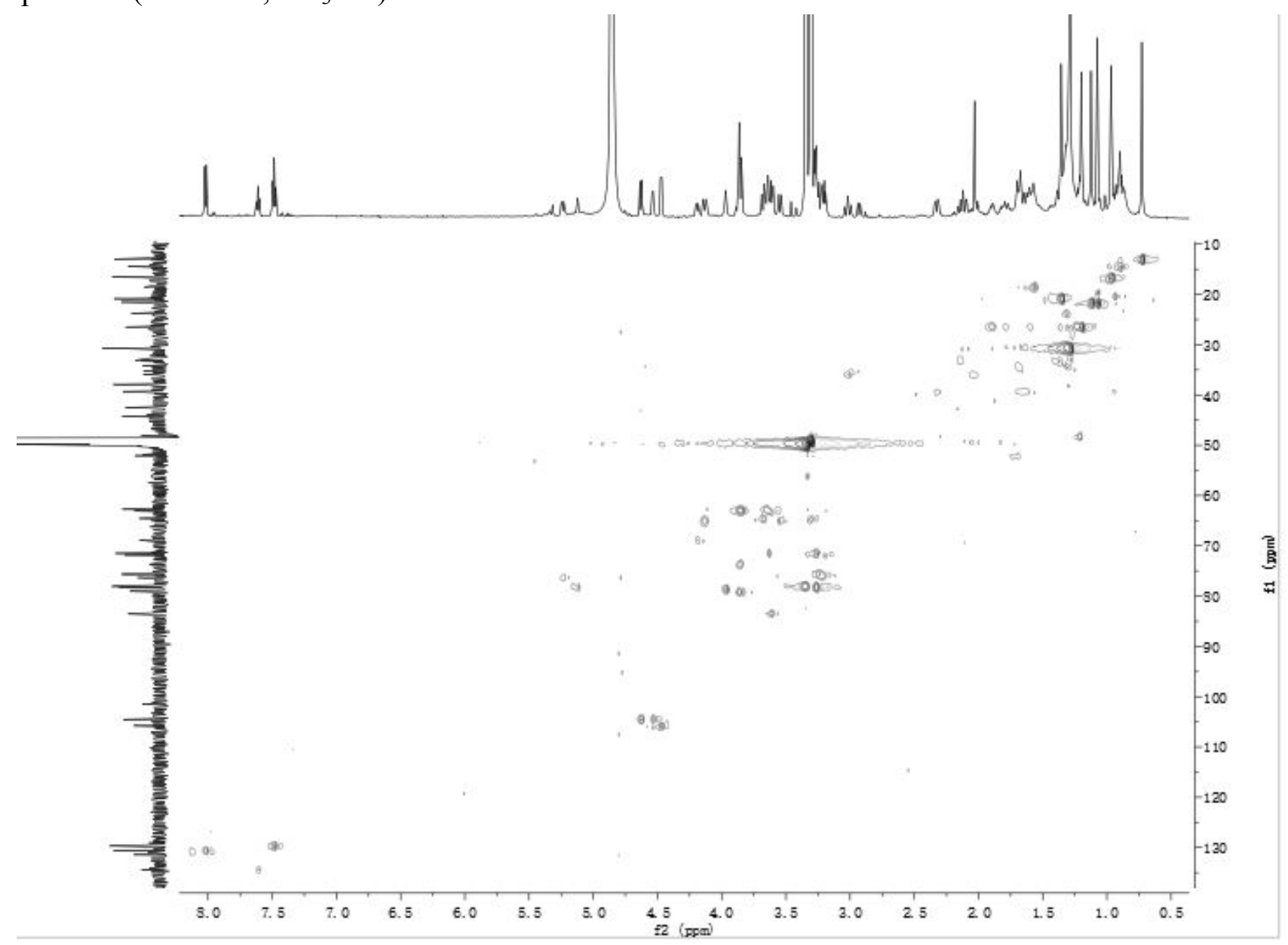


Compound 7: ${ }^{1} \mathrm{H}-{ }^{1} \mathrm{H}$ COSY spectrum $\left(600 \mathrm{MHz}, \mathrm{CD}_{3} \mathrm{OD}\right)$

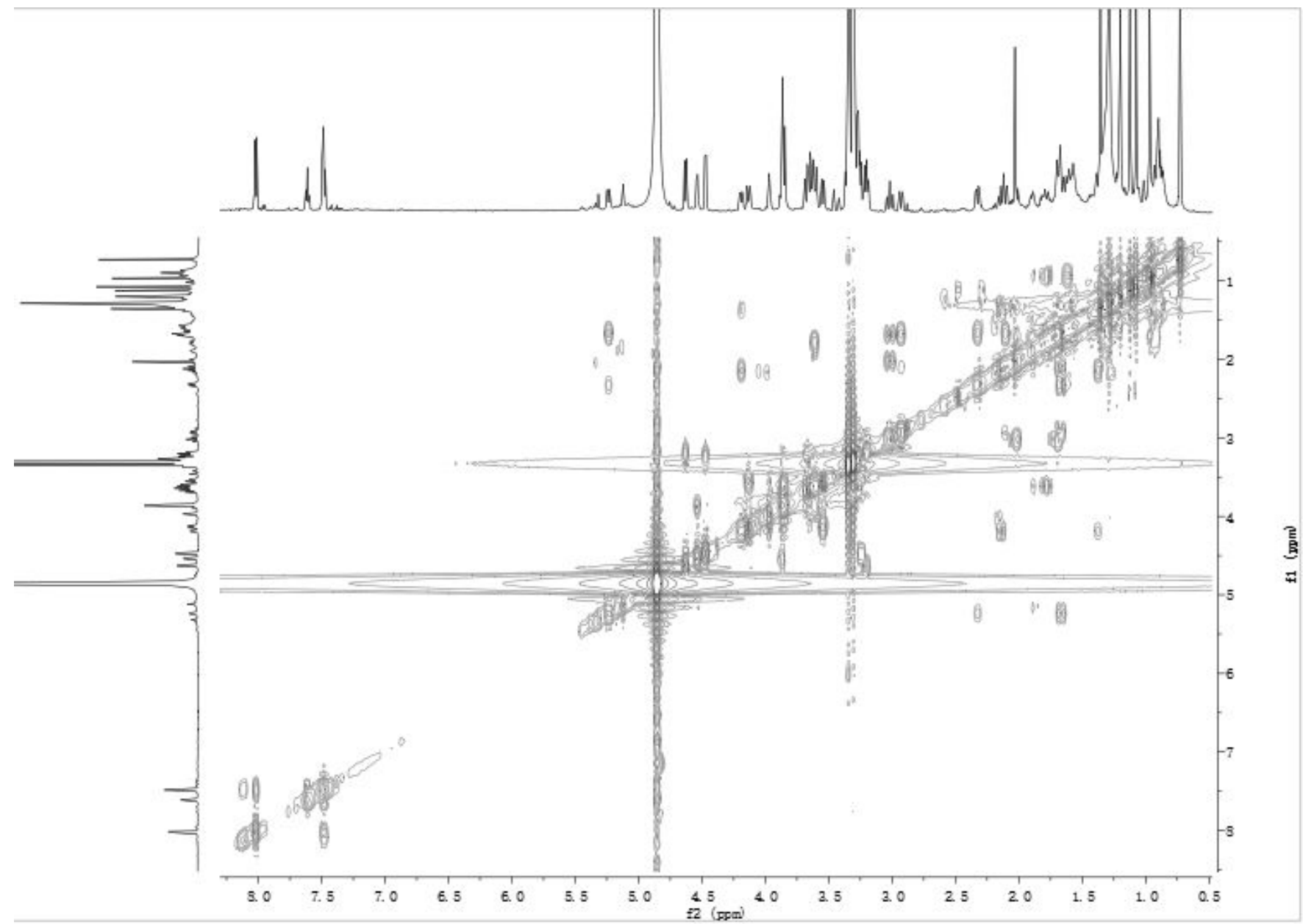


Compound 7: $\mathrm{HMBC}$ spectrum (600 MHz, $\left.\mathrm{CD}_{3} \mathrm{OD}\right)$

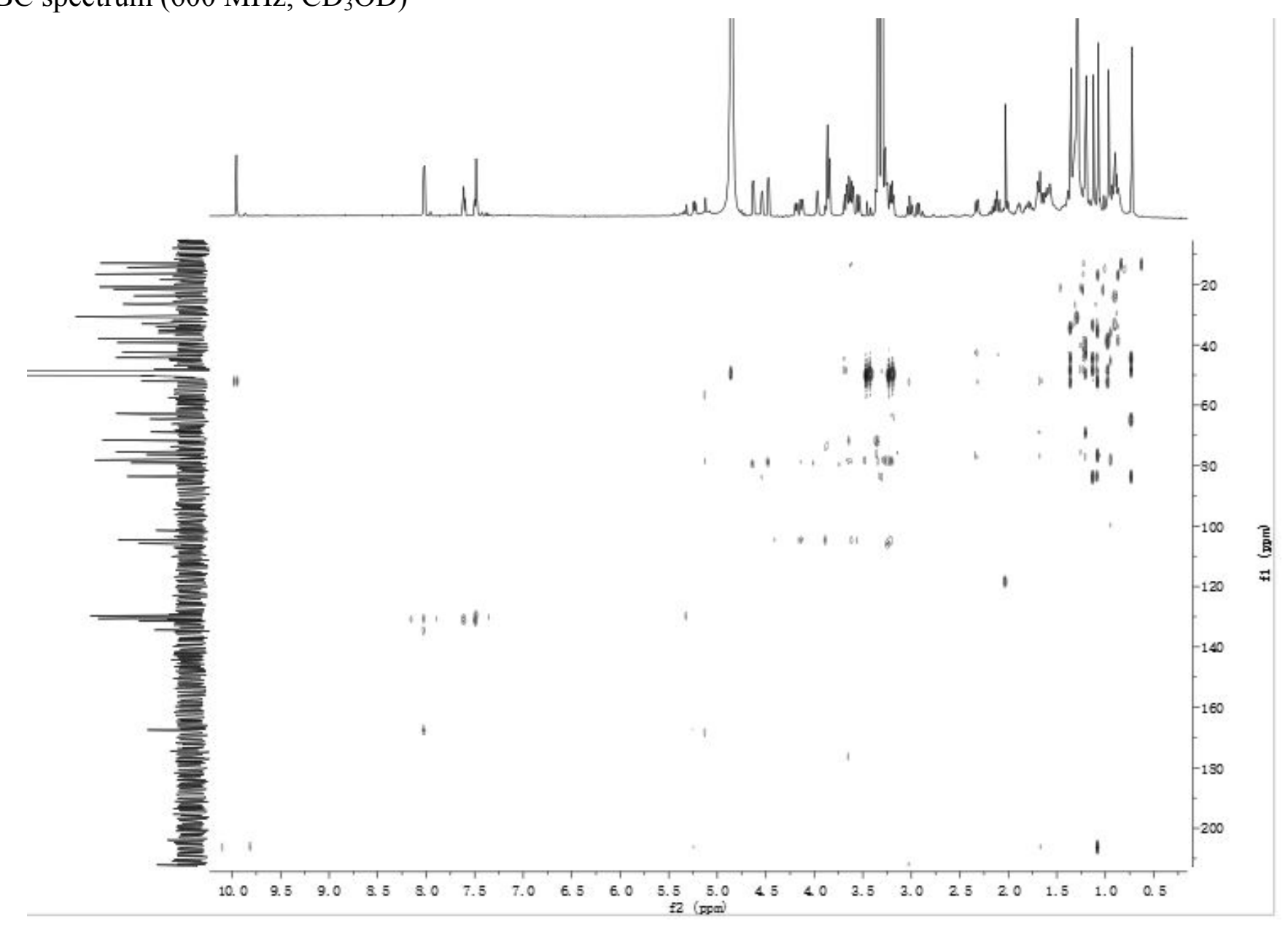


Compound 7: NOESY spectrum (600 MHz, $\left.\mathrm{CD}_{3} \mathrm{OD}\right)$

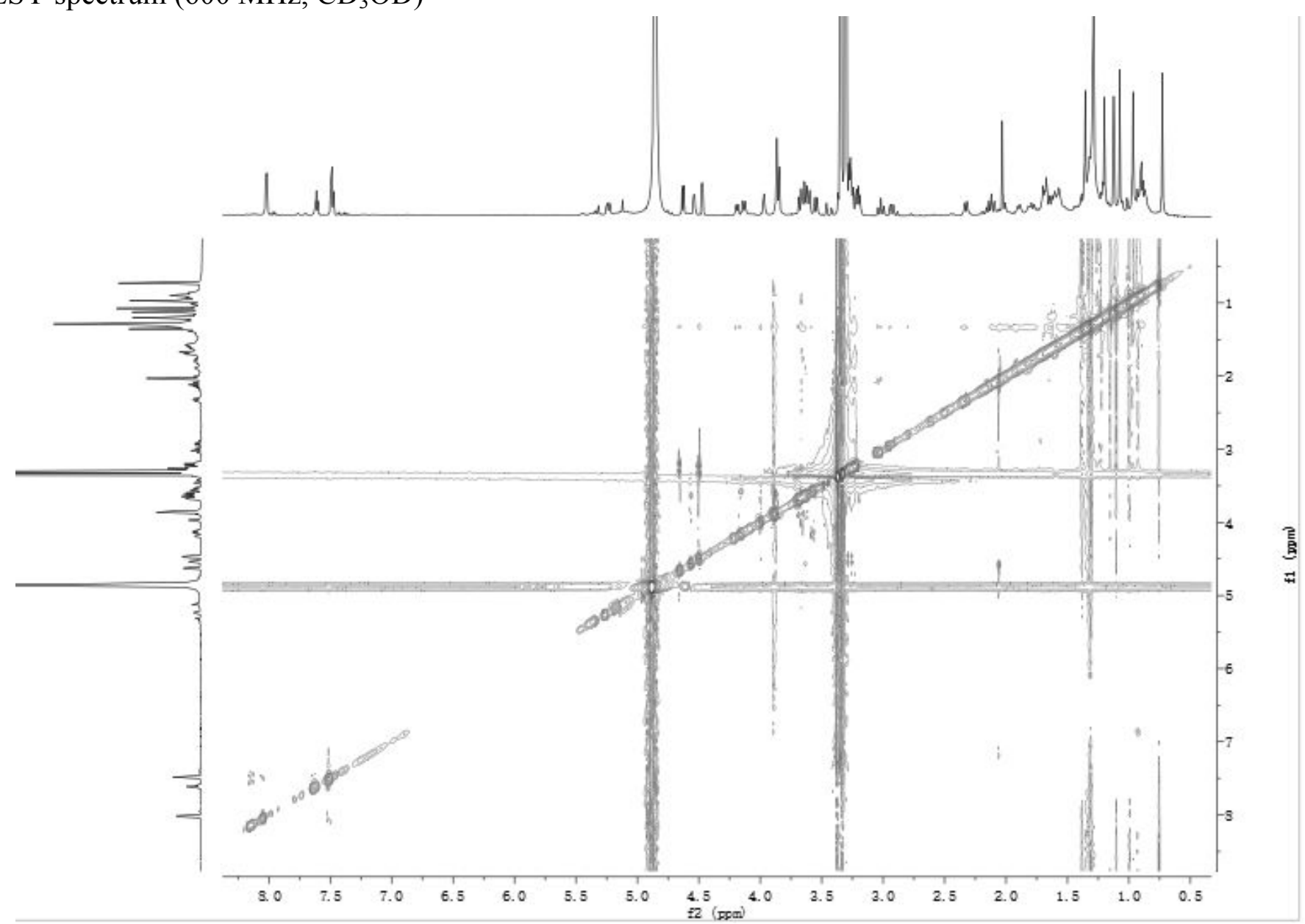


Compound 8: ${ }^{1} \mathrm{H}$ NMR spectrum $\left(600 \mathrm{MHz}, \mathrm{CD}_{3} \mathrm{OD}\right)$

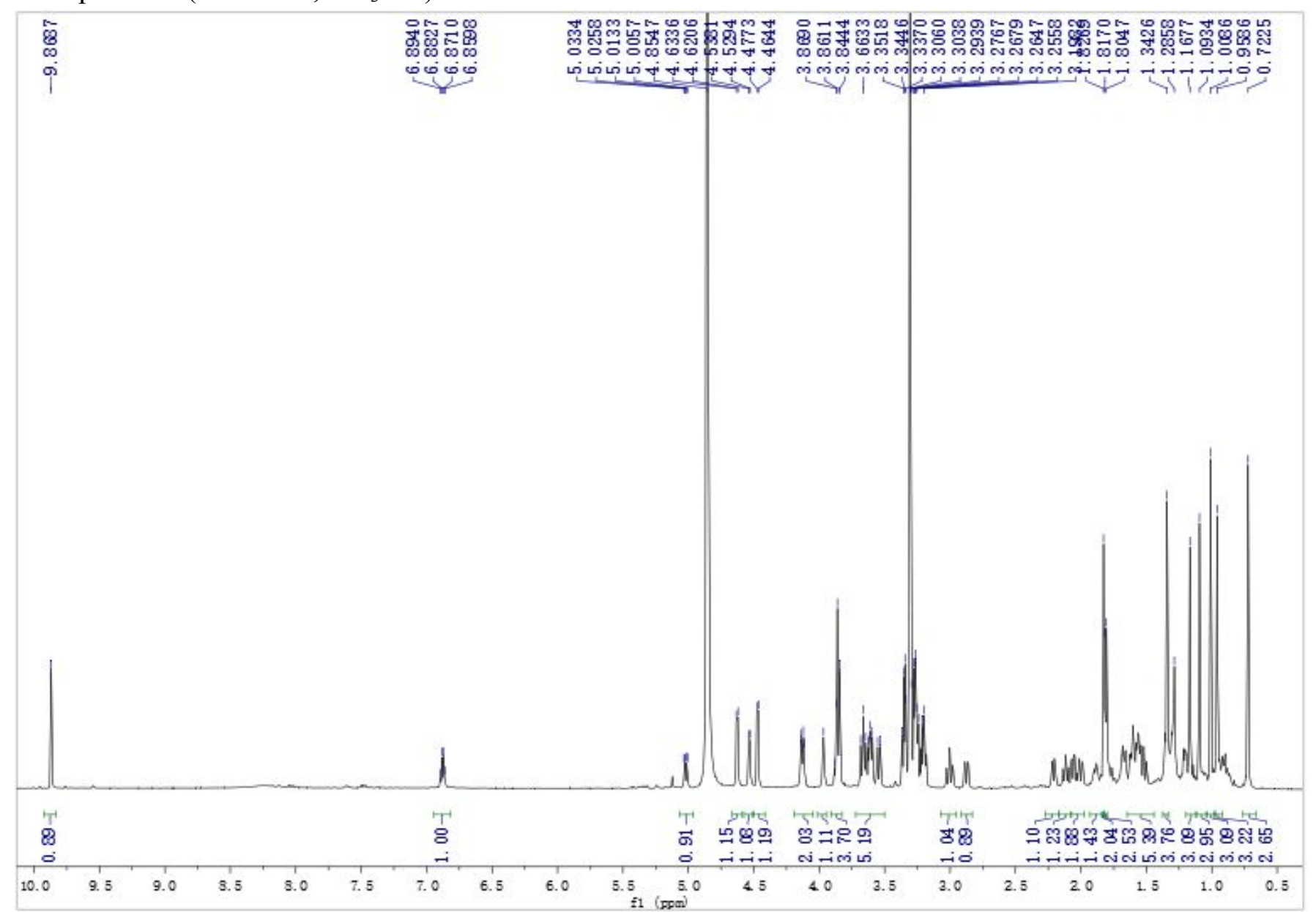


Compound 8: ${ }^{13} \mathrm{C}$ NMR spectrum $\left(600 \mathrm{MHz}, \mathrm{CD}_{3} \mathrm{OD}\right)$

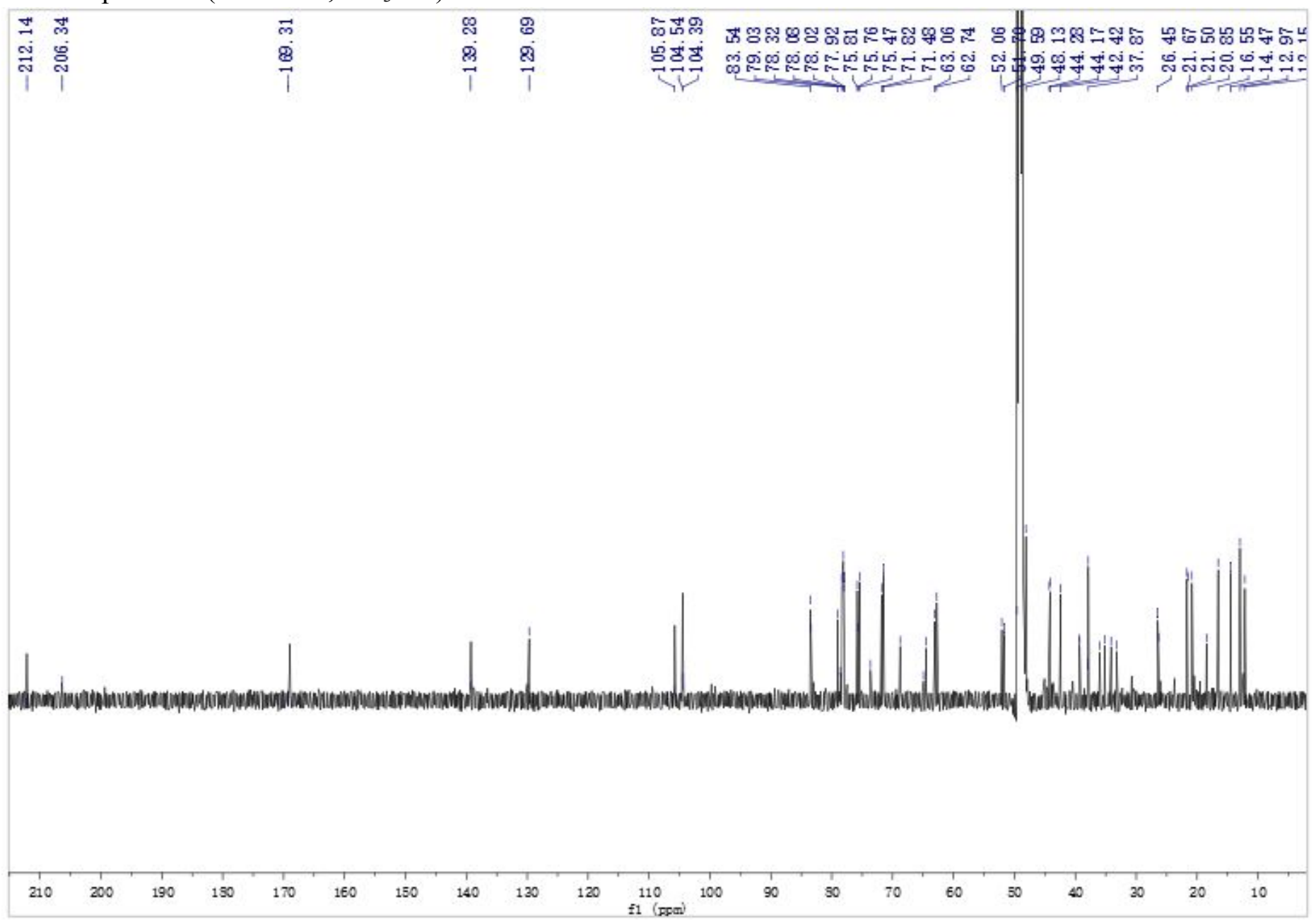


Compound 8: HSQC spectrum (600 MHz, $\mathrm{CD}_{3} \mathrm{OD}$ )

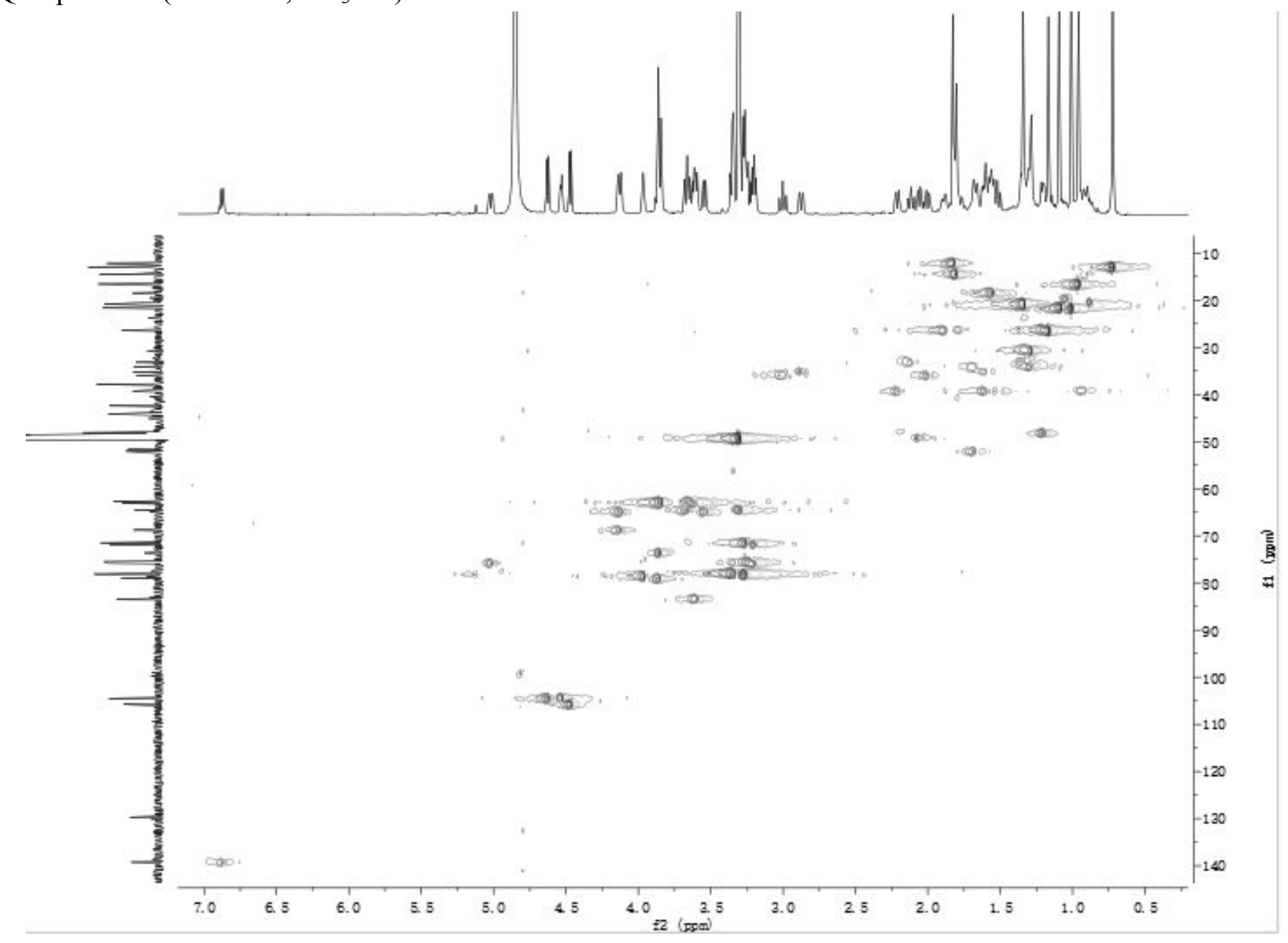

43 
Compound 8: ${ }^{1} \mathrm{H}-{ }^{1} \mathrm{H}$ COSY spectrum $\left(600 \mathrm{MHz}, \mathrm{CD}_{3} \mathrm{OD}\right)$

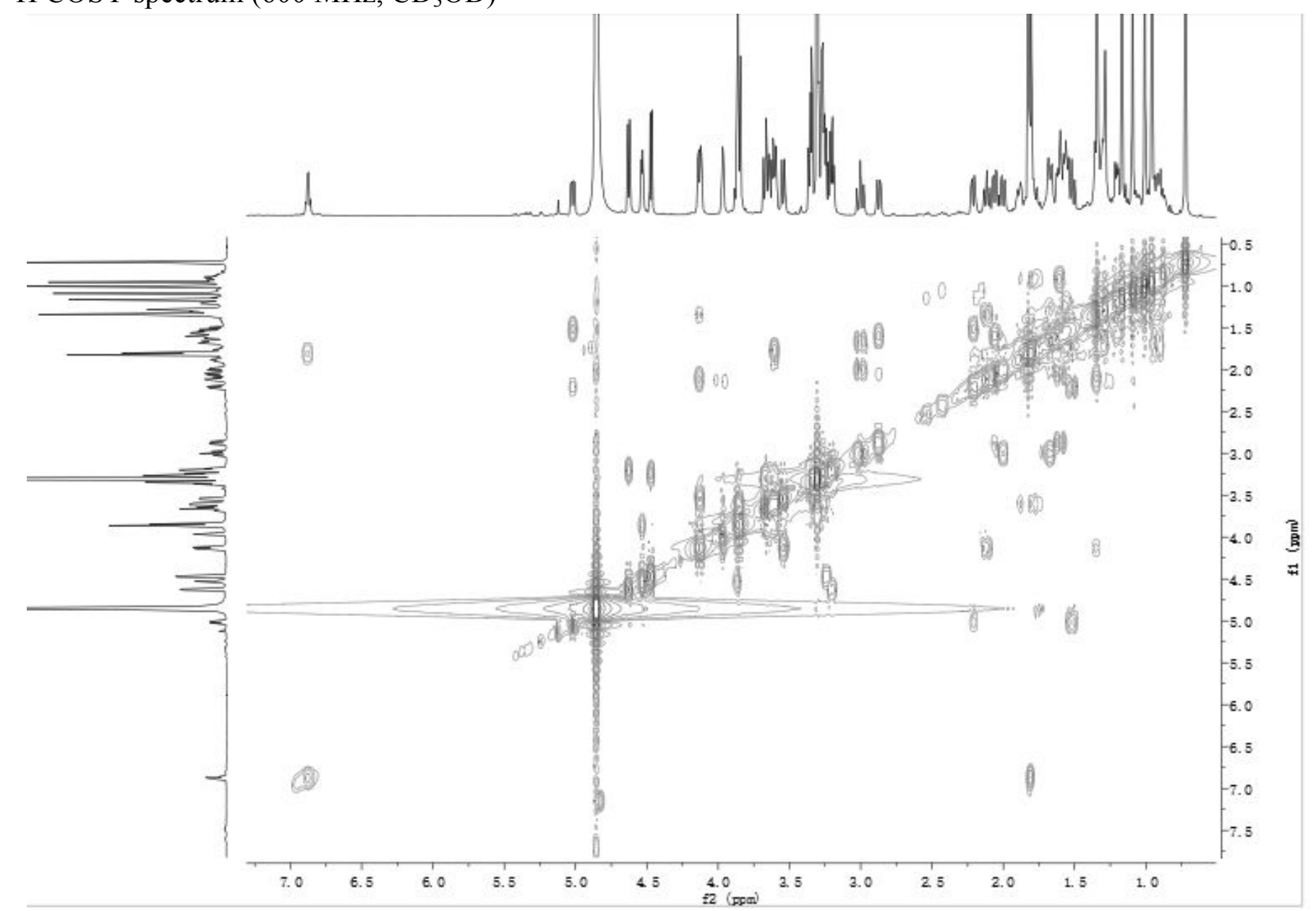


Compound 8: $\mathrm{HMBC}$ spectrum (600 MHz, $\left.\mathrm{CD}_{3} \mathrm{OD}\right)$

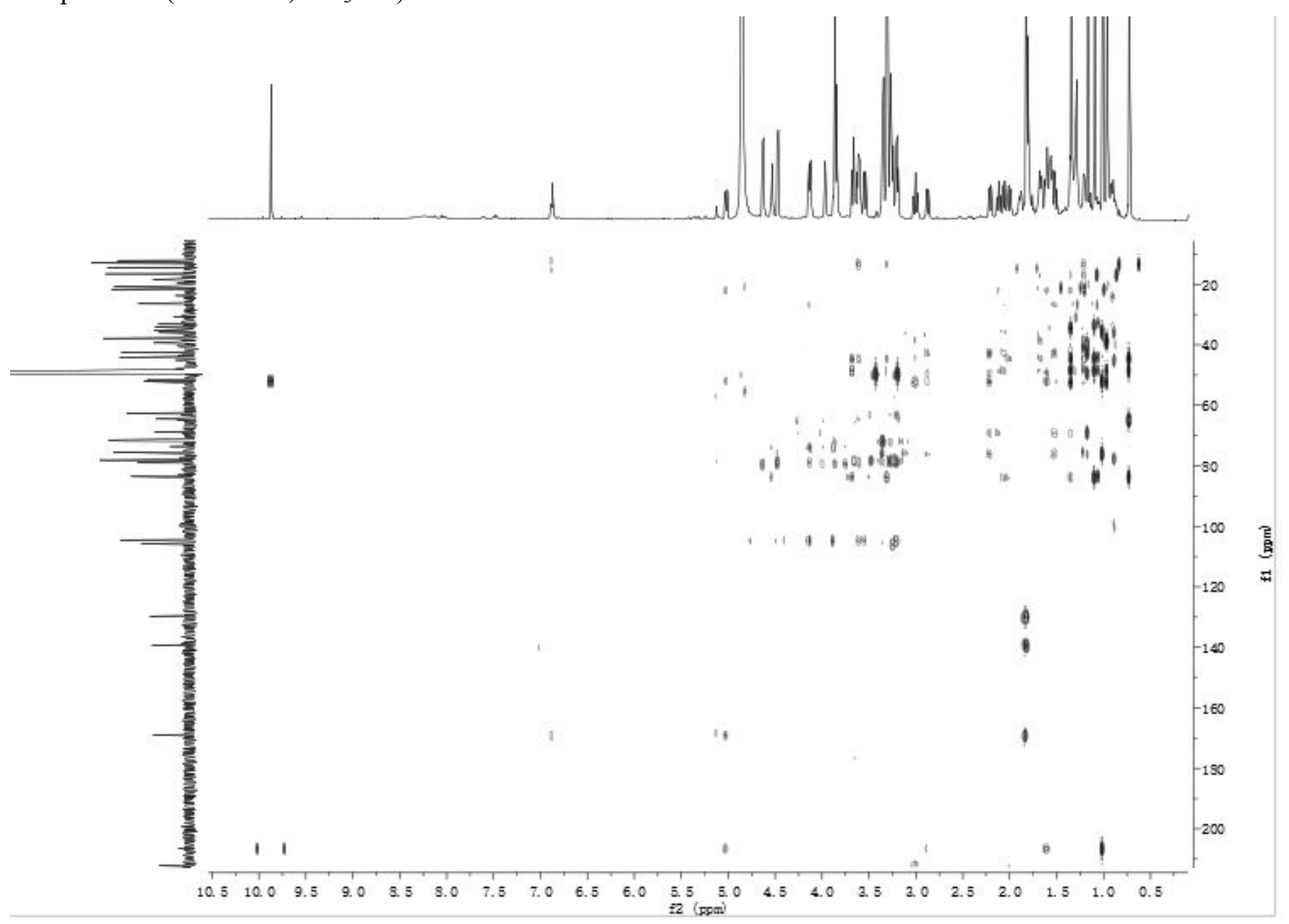


Compound 8: NOESY spectrum (600 MHz, $\left.\mathrm{CD}_{3} \mathrm{OD}\right)$

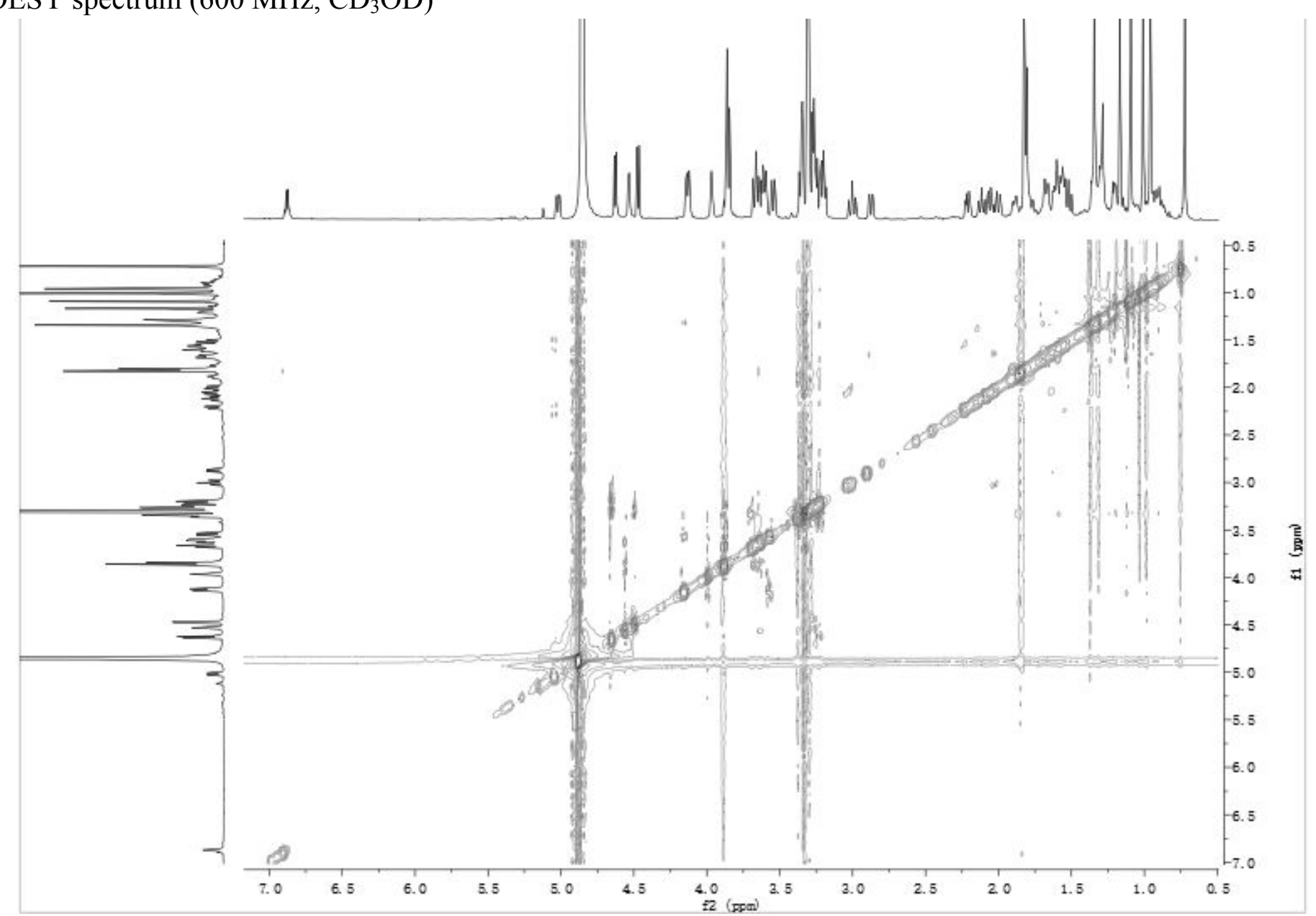

